Article

\title{
Geology and Isotope Systematics of the Jianchaling Au Deposit, Shaanxi Province, China: Implications for Mineral Genesis
}

\author{
Su-Wei Yue ${ }^{1,2, *}$, Deng-Feng Li ${ }^{3,4, *}$, Leon Bagas ${ }^{5,6}$, Jing Fang ${ }^{7}$ and Zhen-Wen Lin ${ }^{8}$ \\ 1 School of Jewellery, Guangzhou College South China University of Technology, Guangzhou 510800, China \\ 2 Key Laboratory of Mineralogy and Metallogeny, Guangzhou Institute of Geochemistry, Chinese Academy of \\ Sciences, Guangzhou 510640, China \\ 3 School of Marine Sciences, Sun Yat-sen University, Guangzhou 510006, China \\ 4 Guangdong Provincial Key Laboratory of Marine Resources and Coastal Engineering, Guangzhou 510006, \\ China \\ 5 Centre for Exploration Targeting, The University of Western Australia, Crawley, WA 6009, Australia; \\ leon.bagas@uwa.edu.au \\ 6 MLR Key Laboratory of Metallogeny and Mineral Assessment, Institute of Mineral Resources, CAGS, \\ Beijing 100037, China \\ 7 Institute of Deep-Sea scicence and Engineering, CAS Sanya 572000, China; fangj@idsse.ac.cn \\ 8 Guangdong Institute of Marine Geological Survey, Guangzhou 510080, China; linzhw9@mail.sysu.edu.cn \\ * Correspondence: Doctor-Yue@foxmail.com (S.-W.Y.); lidf@mail.sysu.edu.cn (D.-F.L.)
}

Received: 9 March 2018; Accepted: 29 March 2018; Published: 3 April 2018

\begin{abstract}
The giant Jianchaling $\mathrm{Au}(52 \mathrm{t} \mathrm{Au})$ deposit is located in the Mian-Lue-Yang Terrane in the southern part of the Qinling Orogen of central China and is hosted by metamorphosed carbonate rocks of the Late Neoproterozoic Duantouya Formation. The deposit consists of multiple generations of mineralised quartz(-carbonate) veins in WNW-trending extensional ductile-brittle shear zones. Based on the mineral assemblages and cross-cutting relationships between the quartz(-carbonate) veins, the paragenesis is characterised by an early coarse-grained pyrite-pyrrhotite-pentlandite-dolomite-quartz assemblage (I), followed by pyrite-sphalerite-galena-carbonate-arsenopyrite-fuchsite-carbonate-quartz containing gold (II), and fine-grained pyrite-dolomite-calcite-quartz-realgar $\left(\mathrm{As}_{2} \mathrm{~S}_{2}\right)$-orpiment $\left(\mathrm{As}_{2} \mathrm{~S}_{3}\right)$ (III). The H-O-C isotope systematics for the three vein sets indicate that the mineralising fluid is probably sourced from the metamorphic dehydration of carbonate rocks in the Duantouya Formation, and gradually mixed with meteoric water during the emplacement of the third vein set. The $\delta^{34} S$ values for sulfides (6.3-16.6\%) from the second auriferous vein set are greater than zero, indicating sulfates reduction from the Neoproterozoic metamorphic rocks (Duantouya Fm). The $\left({ }^{206} \mathrm{~Pb} /{ }^{204} \mathrm{~Pb}\right)$ i ratios from pyrite (17.521-18.477) from each of the vein sets overlap those of the ultramafic rocks (18.324-18.717) and the Bikou Group (17.399-18.417), indicating that the units are possible sources for the sulfides in the mineralisation. Both $\varepsilon N d(t)$ and Isr(t) of sulfide overlap with the meta-ultramafic field and Duantouya formation and dominated with mature Sr-Nd character, which indicated that the Duantouya may play an important role during the ore formation and there may exist a minor ultramafic source that is involved in the ore fluid. The S-Pb-Sr-Nd isotopic ratios are closely related to those of the Bikou Group and Duantouya Formation, which indicates that the mineralised fluid has interacted with both units. Combining the previously published data with data from this study on the mineralised area, we surmise that Jianchaling is characteristic of an orogenic-type gold deposit related to the Triassic Qinling Orogeny associated with continental collision.
\end{abstract}

Keywords: Jianchaling Au deposit; isotope geochemistry; ore genesis; orogenic-type gold deposit; Qinling Orogen; central China 


\section{Introduction}

The different tectonic events are accompanied by different types of mineralisation and associated hydrothermal alteration of different ages [1-3], including orogenic-Au deposits [4-6]. Many of these deposits record multiple deformation events, making then difficult to study and leading to uncertainty in their genesis [7-9]. Nonetheless, some of the deposits have been identified as orogenic-types, such as the Juneau Gold Belt in America [10], the Sukhoi Log gold deposit in Russia [11], the Victorian gold province in SE Australia [12], the Jiaodong gold province in China [13] and the Shanggong, Wenyu and Huachanggou Au deposits, and Tieluping, Weishancheng, Yindonggou, Wangpingxigou, Dahu and Zhifang Ag-Au-Mo deposits [14-26] in China of the Qinling Orogen.

Other deposits present in the Qinling Orogen include porphyry-, skarn-, breccia pipe-, hydrothermal-, Carlin-, and epizonal-types, which have been studied in detail [1,27-38]. Orogenic gold deposits recognised in the orogen include Jianchaling, Lijiagou and Huachanggou [8,25,39], of which Jianchaling is the largest (Figure 1). However, the genesis of the giant Jianchaling Au deposit is still poorly understood.
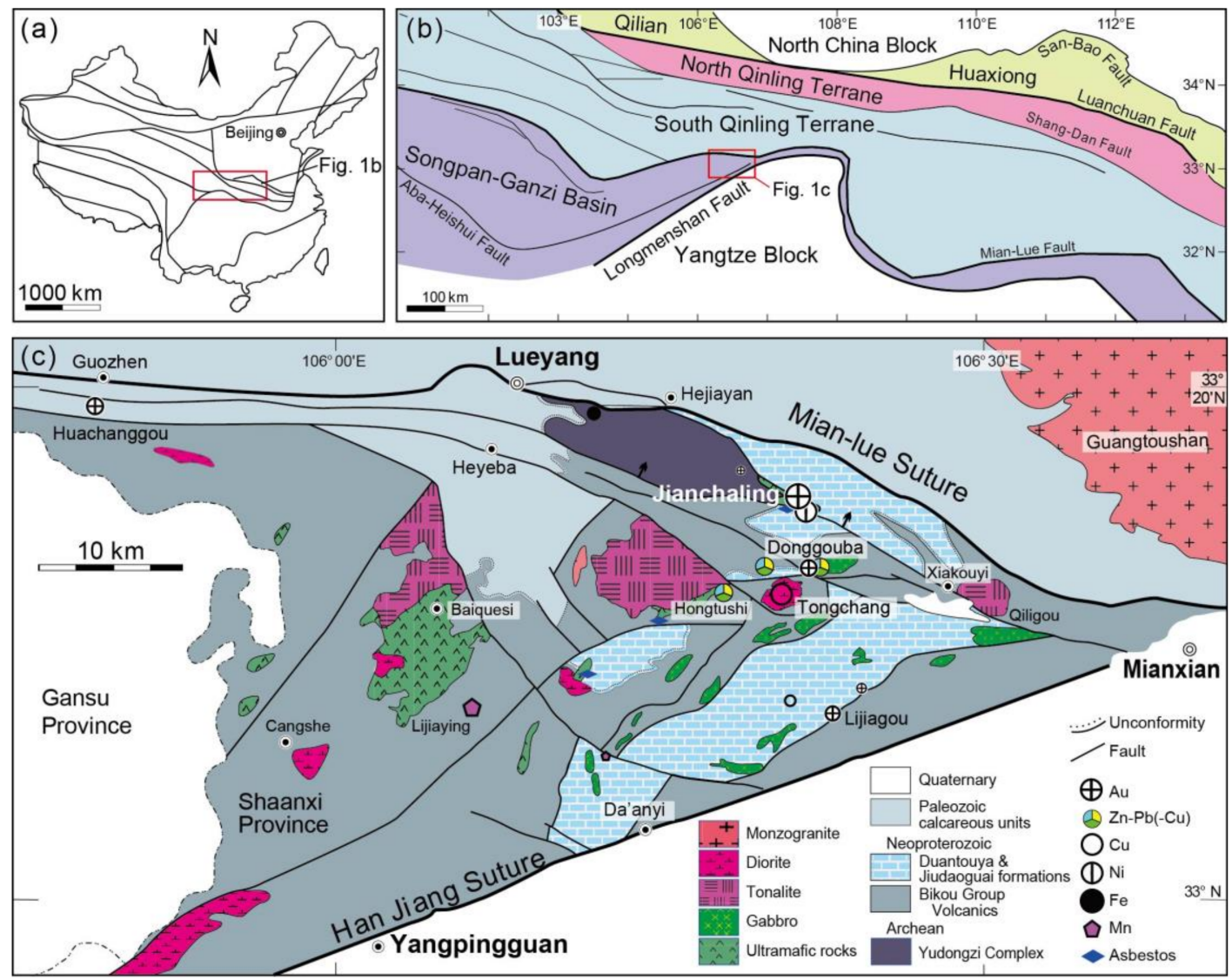

Figure 1. Geological maps showing: (a) the tectonic setting of China; (b) tectonic subdivision of Qinling Orogen and location of the Mianxian, Lueyang, and Yangpingguan (MLY) area [18]; and (c) regional geology and location of gold deposits in the MLY [39].

The Jianchaling Au deposit is located in the triangular area marked by Mianxian, Lueyang, and Yangpingguan towns (MLY), in the South Qinling Terrane of the Qinling Orogen (Figure 1c). The deposit has a measured resource of $52 \mathrm{t} A \mathrm{u}$ with an average grade of $5 \mathrm{~g} / \mathrm{t}$ hosted by quartz-veins in a ductile-brittle shear zone [21,39-43]. Even though the genesis of the mineralisation at the deposit has been studied, the source of the metals and nature of the mineralising fluids have remained unclear. 
This contribution summarises the geology, mineralisation, and $\mathrm{H}-\mathrm{O}-\mathrm{C}-\mathrm{S}$ stable and $\mathrm{Pb}-\mathrm{Sr}-\mathrm{Nd}$ isotopic systematics for the giant Jianchaling deposit with the aim to clarify the source of the metals and nature of the mineralising fluid. This, in turn, provides a better understanding of the tectonic and mineralising processes in MLY.

\section{Geological Setting}

The Qinling Orogen is located south of the North China Block (NCB) and has experienced a complex tectonic history [44-46]. It is located in the central portion of the east-trending Central China Orogen, and developed during the Mesozoic collision between the North China and Yangtze blocks (Figure 1a,b) [1,47-49]. The Luanchuan Fault is the northern boundary of the orogen, and the southern boundary is the Longmenshan Fault. Deformation associated with the orogen extends into the Huaxiong Domain that is also included in the southern part of the Precambrian North China Block. The subdivisions of the orogen are shown in Figure $1 b$.

The Jianchaling deposit is a fault-controlled lode-gold deposit hosted by the Neoproterozoic Duantouya Fm in the eastern part of the MLY (Figure 1). The group unconformably underlies Late Paleozoic turbiditic successions in the Songpan-Ganzi Basin situated between the Mian-lue Suture to the north and Han Jiang Suture to the south (Figure 1c). The area is an important part of the Qinling Orogen and hosts many mineral deposits (e.g., $\mathrm{Au}, \mathrm{Cu}, \mathrm{Ni}, \mathrm{Zn}, \mathrm{Pb}, \mathrm{Mn}$ ) in various lithological and structural settings (Figure 1c), and is commonly known as the Golden Triangle [42].

The rock-types in the MLY area include metamorphosed Neoarchean granite-greenstones in the Yudongzi Complex, Neoproterozoic volcanic sequences in the Bikou Group, Late Neoproterozoic calcareous units in the Duantouya and Jiudaoguai formations, and Paleozoic carbonate in the Lueyang Formation (Figure 2). The Bikou Group is widespread in the area and consists of greenschist to amphibolite facies felsic and mafic volcanic protoliths. Various generations of metamorphism and ductile to brittle NE- and NW-trending faults (including thrusts and basin-forming extensional faults) have developed in the area, which control the location of felsic, mafic and ultramafic intrusive rocks and mineralisation (Figure 2).
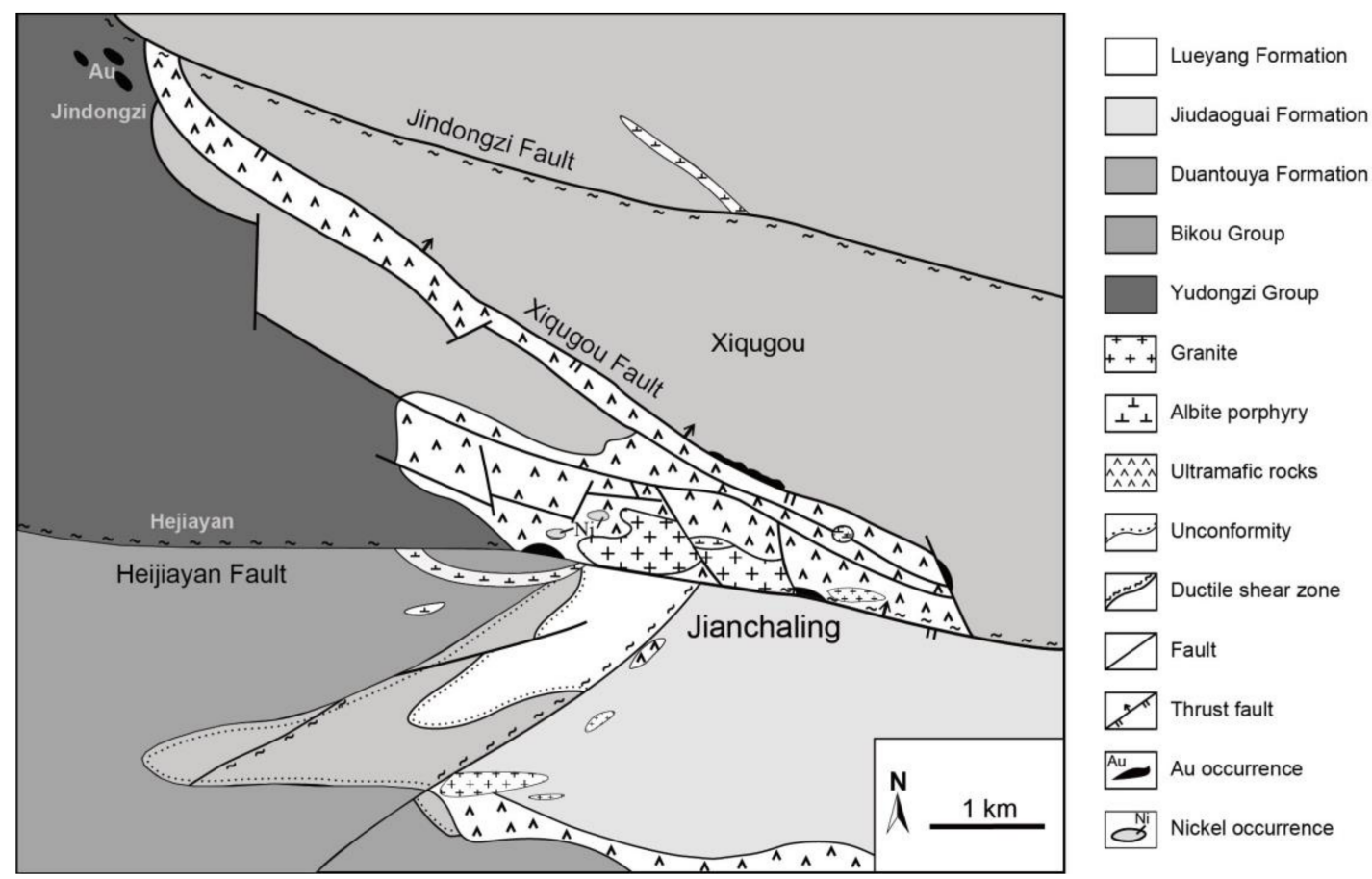

Figure 2. Geological sketch map of the Jianchaling Au deposit [21,39]. 


\section{Geology and Mineralization of the Jianchaling Area}

Rocks outcropping in the Jianchaling area Neoarchean Yudongzi Complex, Neoproterozoic Bikou Group, Late Neoproterozoic Duantouya and Jiudaoguai formations, and Paleozoic Lueyang Group (Figure 2). The deposit can be subdivided into northern and southern zones containing five lenticular mineralisation in the WNW-trending Hejiayan and NW-trending Xiqugou faults and their splays. The richest mineralisation is the "No.1 Au orebody" that is $1950 \mathrm{~m}$ long, up to $23 \mathrm{~m}$ wide, and dips $>43^{\circ} \mathrm{N}$.

The Hejiayan and Xiqugou faults are brittle-ductile shear zones [50], and host mineralised lenticular quartz veins at the contact between ultramafic rocks and calcareous beds in the Late Neoproterozoic Duantouya and Jiudaoguai formations (Figures 2 and 3). The quartz veins shallowly dip between $20^{\circ}$ and $40^{\circ} \mathrm{WSW}$ is places in steeply dipping sections of the fault.

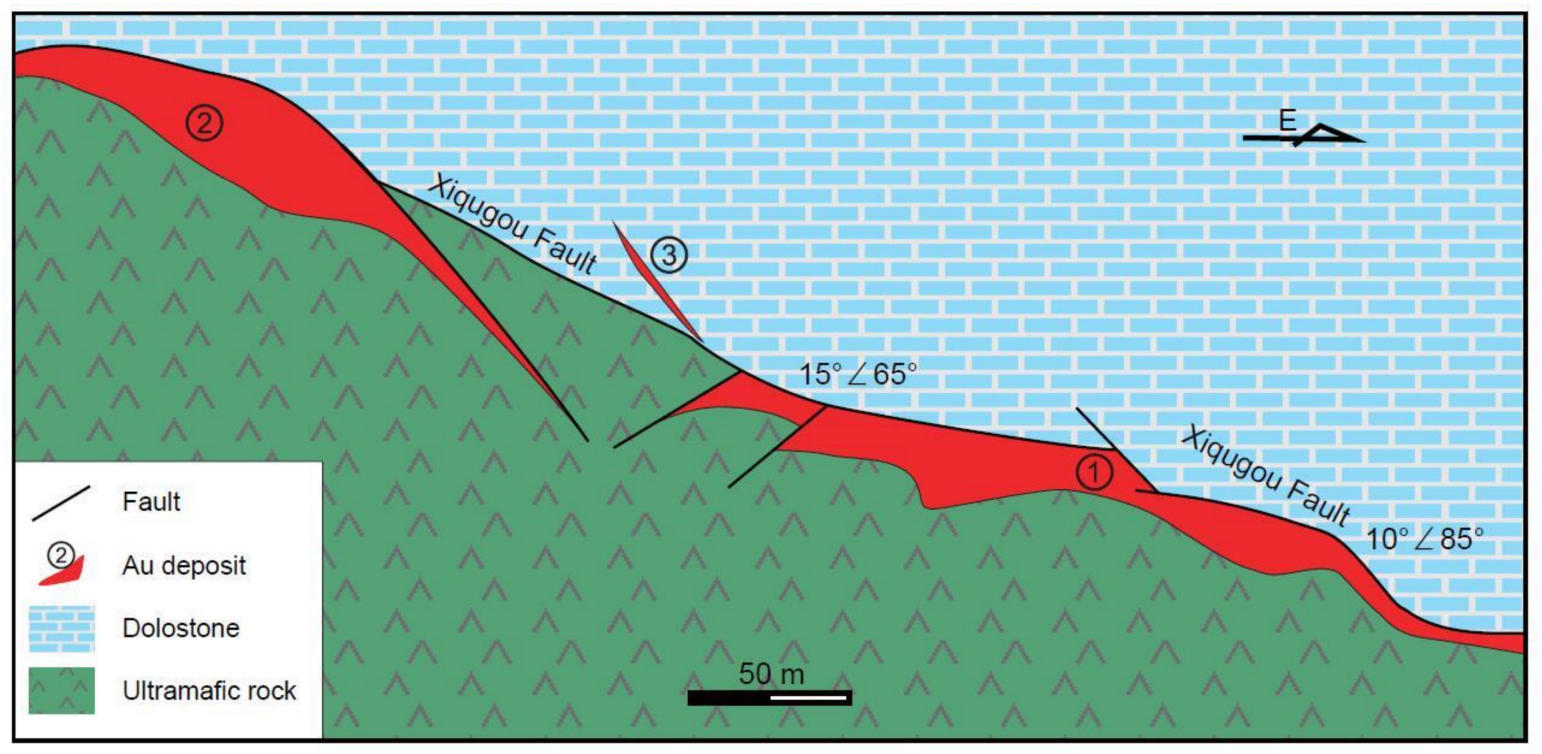

Figure 3. Cross-section of the of the Jianchaling Au deposit at a $870 \mathrm{~m}$ elevation showing the relationship of the mineralisation and fault $[21,51]$.

The mineralisation includes native gold and lesser amounts of electrum and native silver associated with pyrite and minor amounts of hematite, magnetite, limonite, chromite, marcasite, realgar, and orpiment. The gangue minerals are dolomite, calcite and quartz, with minor amounts of serpentine, fuchsite, and albite (Figure $4 a-c)$ [39].

Yue $[21,39]$ recognized early deformed and recrystallised quartz-pyrite-carbonate vein crosscut by the quartz-gold—fuchsite-arsenopyrite—pyrite—-magnetite second generation vein set, and the third generation of dolomite-calcite-quartz-fine-grained pyrite-realgar-orpiment veins (Figure 4c). The pyrite associated with each of the vein sets are also distinct from each other. The first vein set contains pyrite that is $>2 \mathrm{~mm}$ across, variably fractured, anhedral to subhedral in shape, and intergrown with euhedral fuchsite and serpentine (Figure $4 \mathrm{~d}, \mathrm{e}$ ). The second pyrite generation is not deformed, subhedral, 1-2 mm across, and commonly has cores or inclusions of the first generation pyrite, chalcopyrite, and sphalerite (Figure $4 \mathrm{f}, \mathrm{g}$ ). This pyrite is also commonly altered to hematite with martite pseudomorphing magnetite (Figure $4 \mathrm{~h}$ ). The third generation of pyrite is $<10 \mu \mathrm{m}$ across, subhedral to euhedral, and present in alteration zones containing the third vein (Figure 4i). The paragenesis is illustrated in Figure 5. 

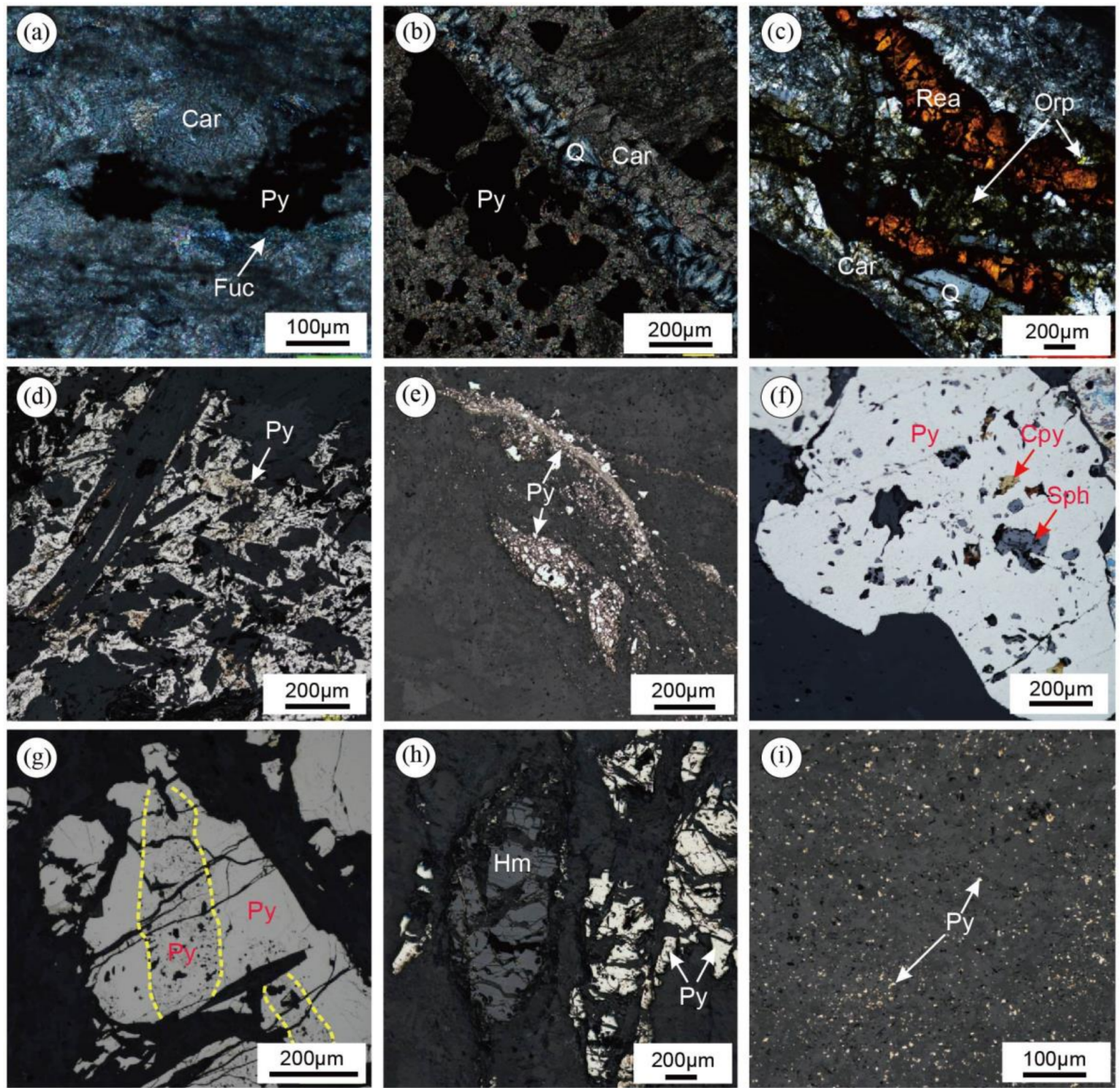

Figure 4. Photomicrographs of samples from the Jianchaling Au eposit showing: (a) intergrown pyrite and fuchsite in the mineralised second vein set; (b) a second vein set with quartz containing pyrite; (c) third vein set with comb-like quartz, calcite and dolomite vein orpiment and realgar; (d) pyrite in the first vein set intergrowth; (e) fine-grained cataclastic pyrite following schistosity developed during brittle-ductile deformation before emplacement of the mineralised second vein set; (f) pyrite wrapping sphalerite and chalcopyrite in the mineralised second vein set; (g) pyrite in the first vein set overgrown by pyrite related to the mineralised second vein set; (h) hematite formed from pyrite, and martite; (i) euhedral fine-grained pyrite in the third vein set. Abbreviations: Cal = calcite; Car = carbonates; $\mathrm{Cpy}=$ chalcopyrite; Dol = dolomite; Fuc = fuchsite; $\mathrm{Hm}=$ hematite; Orp = orpiment; Py = pyrite; $\mathrm{Q}=$ quartz; Rea = realgar; $\mathrm{Sph}=$ sphalerite. 


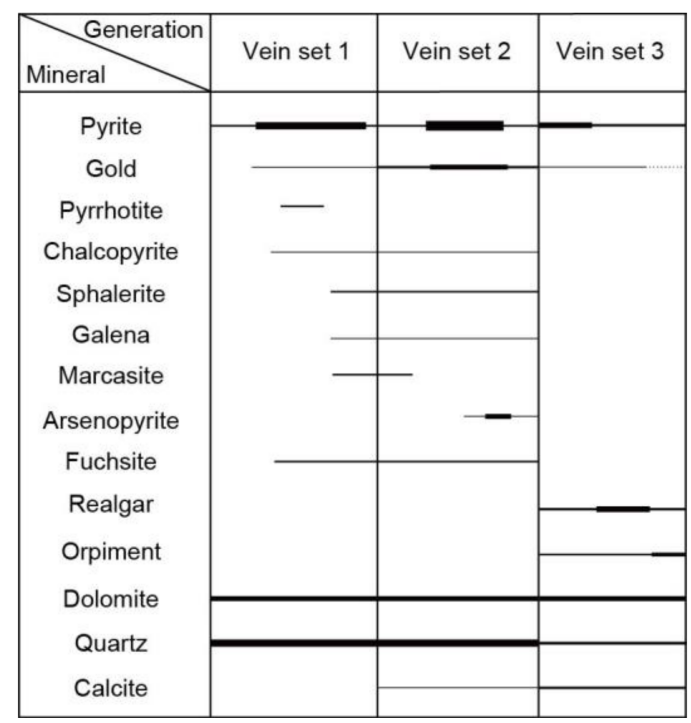

Figure 5. Paragenetic relationship of the minerals at the Jianchaling Au deposit [21].

\section{Sampling and Analytical Methods}

Fresh samples were collected from each of the vein sets at depth in the Jianchaling mine. The samples were examined in thin section to confirm their paragenetic relationship. The sample characteristics are listed in Table 1.

Six samples of pyrite from the second (mineralised) vein set and five samples of the Duantouya Formation and meta-ultramafic from the wall-rock were collected for lead, strontium, and neodymium isotopic studies. The samples were crushed into power.

The samples were then crushed to a minus 10 mesh size (420 microns, and sulfide and carbonate fragments were handpicked under a binocular microscope. Between 10 and $50 \mathrm{mg}$ of the powder was leached in acetone and washed in distilled and deionised water to remove contamination, then dried in an oven at $60{ }^{\circ} \mathrm{C}$. The samples were then dissolved in a solution of $\mathrm{HF}+\mathrm{HNO}_{3}+\mathrm{HClO}_{4}$, dried, and redissolved in $6 \mathrm{~N} \mathrm{HCl}$, redried, and redissolved in $0.5 \mathrm{~N} \mathrm{HCl}$ (for $\mathrm{Sr}$ and Nd separation) or $0.5 \mathrm{~N}$ $\mathrm{HBr}$ (for $\mathrm{Pb}$ separation). The $\mathrm{Sr}$ and $\mathrm{Nd}$ fractions were separated following standard chromatographic techniques using AG50 $\times 8$ and PTFE-HDEHP resins with $\mathrm{HCl}$ as eluent. The $\mathrm{Pb}$ fraction was separated using a strong alkali anion exchange resin with $\mathrm{HBr}$ and $\mathrm{HCl}$ as eluents.

The lead isotopes were analysed using a MAT-261 thermal ionization mass spectrometer with the standard NBS 981 in the Analytical Laboratory at the Beijing Research Institute of Uranium Geology, China. Measurements of the common- $\mathrm{Pb}$ standard NBS 981 gave average ${ }^{208} \mathrm{~Pb} /{ }^{206} \mathrm{~Pb}$ values of $2.1681 \pm 0.0008,{ }^{207} \mathrm{~Pb} /{ }^{206} \mathrm{~Pb}$ of $0.91464 \pm 0.00033$, and ${ }^{204} \mathrm{~Pb} /{ }^{206} \mathrm{~Pb}$ values of $0.059042 \pm 0.000037$; the uncertainty is of $<0.1 \%$ at the $95 \%$ confidence level. Some of the $\mathrm{U}$, Th and $\mathrm{Pb}$ values were used to estimate the $\mathrm{Pb}$ isotope ratios assuming an age of $198 \mathrm{Ma}$, which is the ${ }^{40} \mathrm{Ar} /{ }^{39} \mathrm{Ar}$ date determined on fuchsite from the Jianchaling deposit [39]. These $\mathrm{Pb}$ isotopic ratios are presented as $\left({ }^{208} \mathrm{~Pb} /{ }^{204} \mathrm{~Pb}\right)_{\mathrm{i}}$, $\left({ }^{207} \mathrm{~Pb} /{ }^{204} \mathrm{~Pb}\right)_{\mathrm{i}}$ and $\left({ }^{206} \mathrm{~Pb} /{ }^{204} \mathrm{~Pb}\right)_{\mathrm{i}}$.

A TRITON thermal ionization mass spectrometer was used to measure the $\mathrm{Sr}$ and $\mathrm{Nd}$ isotopes in the Analytical Laboratory at the Tianjin Institute of Geology and Mineral Resources, China. The ${ }^{87} \mathrm{Sr} /{ }^{86} \mathrm{Sr}$ isotope ratios were normalized against the ${ }^{86} \mathrm{Sr} /{ }^{88} \mathrm{Sr}=0.1194$ and ${ }^{143} \mathrm{Nd} /{ }^{144} \mathrm{Nd}$ isotope ratios to ${ }^{146} \mathrm{Nd} /{ }^{144} \mathrm{Nd}=0.7219$. The JNdi Nd-Standard yielded ${ }^{143} \mathrm{Nd} /{ }^{144} \mathrm{Nd}$ ratios of $0.512118 \pm 0.3$ against a reference value of $0.512115 \pm 0.7$ [52], and the NBS $987 \mathrm{Sr}$ standard with a reference level of 0.710248 was used yielding ${ }^{87} \mathrm{Sr} /{ }^{86} \mathrm{Sr}$ ratios of $0.710250 \pm 0.7$. The $\mathrm{Sr}$ and $\mathrm{Nd}$ isotopic compositions were measured with a thermal ionization ISOPROBE-T mass spectrometer. 
Table 1. The geological characteristics of samples from the Jianchaling Au deposit (modified from [21]).

\begin{tabular}{|c|c|c|c|c|}
\hline Sample NO. & Mineral & Sample Description & Stage & Analyzed Isotope \\
\hline $800-53-2$ & Dolomite & Dolomite veins in the Serpentinite with shear deformation & 1 & $\mathrm{C}, \mathrm{O}$ \\
\hline $860-52-7$ & Dolomite & Dolomite veins in the Serpentinite with shear deformation & 1 & $\mathrm{C}, \mathrm{O}$ \\
\hline $\mathrm{J}-6$ & Dolomite & Pale green dolomite veins in the cataclastic dolomite & 1 & $\mathrm{C}, \mathrm{O}$ \\
\hline $913-32-7$ & Calcite & Calcite veins in the cataclastic dolomite with a small number of pyrites & 2 & $\mathrm{C}, \mathrm{O}$ \\
\hline $913-32-6$ & Calcite & Calcite veins in the cataclastic dolomite with a small number of pyrites & 2 & $\mathrm{C}, \mathrm{O}$ \\
\hline 913-1 & Dolomite & Milky white dolomite vein & 3 & $\mathrm{C}, \mathrm{O}$ \\
\hline $913-2$ & Dolomite & Milky white dolomite vein & 3 & $\mathrm{C}, \mathrm{O}$ \\
\hline $960-38-1$ & Dolomite & Milky white dolomite vein & 3 & $\mathrm{C}, \mathrm{O}$ \\
\hline $860-51-5$ & Dolomite & Dolomite veins in the listwanite with a small number of pyrites & 3 & $\mathrm{C}, \mathrm{O}$ \\
\hline $860-51-6$ & Dolomite & Dolomite veins in the listwanite with a small number of pyrites & 3 & $\mathrm{C}, \mathrm{O}$ \\
\hline hjy-8 & Pyrite & Grain cluster pyrite with shear deformation & 1 & $\mathrm{~S}, \mathrm{~Pb}$ \\
\hline $\mathrm{J} 3$ & Pyrite & Massive sulfide ore & 2 & $\mathrm{~S}, \mathrm{~Pb}, \mathrm{Sr}, \mathrm{Nd}$ \\
\hline $\mathrm{J} 3$ & Pyrrhotite & Massive sulfide ore & 2 & $\mathrm{~S}$ \\
\hline hjy-1 & Pyrite & Silicified and fuchsite dolomite ore rocks with disseminated pyrites & 2 & $\mathrm{~S}, \mathrm{~Pb}, \mathrm{Sr}, \mathrm{Nd}$ \\
\hline hjy-12 & Pyrite & Silicified and fuchsite dolomite ore rocks with disseminated pyrites & 2 & $\mathrm{~S}, \mathrm{~Pb}, \mathrm{Sr}, \mathrm{Nd}$ \\
\hline hjy-10 & Pyrite & Intensive silicified and fuchsite dolomite ore rocks with disseminated pyrites & 2 & $\mathrm{~S}, \mathrm{~Pb}, \mathrm{Sr}, \mathrm{Nd}$ \\
\hline hjy-20 & Pyrite & Silicified and fuchsite dolomite ore rocks with disseminated pyrites & 2 & $\mathrm{~S}, \mathrm{~Pb}, \mathrm{Sr}, \mathrm{Nd}$ \\
\hline hjy-14 & Pyrite & Weak silicified and fuchsite dolomite ore rocks with disseminated pyrites & 2 & $\mathrm{~S}, \mathrm{~Pb}, \mathrm{Sr}, \mathrm{Nd}$ \\
\hline $\mathrm{H}-4$ & Whole-rock & Shale (Duantouya Formation) & & $\mathrm{S}, \mathrm{Pb}, \mathrm{Sr}, \mathrm{Nd}$ \\
\hline $960-34-1$ & Whole-rock & Dolomite & & $\mathrm{S}, \mathrm{Pb}, \mathrm{Nd}$ \\
\hline $960-28-1$ & Whole-rock & Dolomite & & $\mathrm{S}, \mathrm{Pb}, \mathrm{Sr}, \mathrm{Nd}$ \\
\hline $780-50-3$ & Whole-rock & Serpentinite & & $\mathrm{S}, \mathrm{Pb}, \mathrm{Sr}, \mathrm{Nd}$ \\
\hline $800-52-1$ & Whole-rock & Listwanite & & $\mathrm{S}, \mathrm{Pb}, \mathrm{Sr}, \mathrm{Nd}$ \\
\hline
\end{tabular}




\section{Isotope Results}

The following are isotopic values determined from samples from the Jianchaling deposit.

\subsection{Carbon, Oxygen and Hydrogen Isotopes}

The $\delta^{13} \mathrm{C}, \delta^{18} \mathrm{O}$ and $\delta \mathrm{D}$ values from quartz and carbonate from each of the three vein sets and the surroundings rocks are listed in Table 2. These results are published on [21]. The $\delta^{13} \mathrm{C}$ values for first vein set ranges from -4.4 to $0.6 \%$, the $\delta^{13} \mathrm{C}$ values for the second (mineralised) vein set ranges from -2.9 to $-0.4 \%$, and the $\delta^{13} \mathrm{C}$ value for the third vein set is between -4.4 to $2.2 \%$ (Table 2).

The $\delta^{18} \mathrm{O}$ values from carbonate and quartz for the first vein set forms two groups of $18.9-23.4 \%$ and $14.0-17.3 \%$. The $\delta^{18} \mathrm{O}$ values from the second vein set are between 13.8 and $19.0 \%$ (averaging 16.4\%), and for the third vein set are between 11.9 and 16.3\% (Table 2).

\subsection{Sulfur Isotopes}

The $\delta^{34} \mathrm{~S}$ isotopic values for the sulfides samples are listed in Table 3 and cited from [21]. The Jianchaling deposit is characterized by highly $\delta^{34} S$ positive values with a narrow range. The first vein set has a $\delta^{34} S$ value of $14.3 \%$ (Table 3). The second vein set has $\delta^{34} S$ values between 8.2 and $14.3 \%$. One shale rock sample of the Duantouya Formation have $\delta^{34} S$ values of $16.6 \%$. Two samples of the metamorphosed ultramafic rocks (listwanite and serpentinite) have $\delta^{34} S$ values of 6.1 to $8.6 \%$.

\subsection{Lead Isotopes of Sulfides and Wall Rocks}

The $\mathrm{Pb}$ isotopic analyses completed for this study and previous data are listed in Table 4 . In order to compare the contribution of the wall rocks, we also put the lead isotopes of the wall rocks including Yudongzi Fm, Porphyritic granite, Duantouya Fm and Bikou Group in Table 3. Sulfides from gold deposits have ${ }^{206} \mathrm{~Pb} /{ }^{204} \mathrm{~Pb}$ values between 17.257 and $18.477,{ }^{207} \mathrm{~Pb} /{ }^{204} \mathrm{~Pb}$ values between 15.530 and 15.704 , and ${ }^{208} \mathrm{~Pb} /{ }^{204} \mathrm{~Pb}$ values of 36.927 to 38.757 (Table 4). Their calculated $\left({ }^{206} \mathrm{~Pb} /{ }^{204} \mathrm{~Pb}\right){ }_{\mathrm{i}}$, $\left({ }^{207} \mathrm{~Pb} /{ }^{204} \mathrm{~Pb}\right)_{\mathrm{i}}$ and $\left({ }^{208} \mathrm{~Pb} /{ }^{204} \mathrm{~Pb}\right)_{\mathrm{i}}$ values are the same as the measured values due to their very low $\mathrm{U}$ and Th contents. Therefore, the analytical data is interpreted as being reliable and representative of the source for metals in the mineralising fluid. The ultramafic rocks have ${ }^{206} \mathrm{~Pb} /{ }^{204} \mathrm{~Pb}$ values of $17.952-19.193,{ }^{207} \mathrm{~Pb} /{ }^{204} \mathrm{~Pb}$ values of $15.520-15.785$, and ${ }^{208} \mathrm{~Pb} /{ }^{204} \mathrm{~Pb}$ ratios of $36.029-38.920$ (Table 4).

\subsection{Strontium and Neodymium Isotopes of Sulfides and Wall Rocks}

Four pyrite samples from the gold-bearing second generation vein set have $\mathrm{I}_{\mathrm{Sr}}$ values in the range 0.706709-0.715929 (average of 0.711973, Table 5), which is similar to that of the meta-ultramafic rocks. The $\left({ }^{143} \mathrm{Nd} /{ }^{144} \mathrm{Nd}\right)_{\mathrm{i}}$ values of the samples are between 0.511376 and 0.512453 (average of 0.511823 , Table 6), and the $\varepsilon_{\mathrm{Nd}}$ values are between -19.6 and 1.4.

Samples from the Duantouya Formation have $\left({ }^{143} \mathrm{Nd} /{ }^{144} \mathrm{Nd}\right)_{\mathrm{i}}$ values of $0.511865-0.512114$ (average of 0.511953 , Table 6), and $\varepsilon_{\mathrm{Nd}}$ values of -10.1 to -5.3 . The meta-ultramafic samples have $\left({ }^{143} \mathrm{Nd} /{ }^{144} \mathrm{Nd}\right.$ ) $\mathrm{i}$ values of $0.512137-0.512152$ (average of 0.512144 , Table 6), and $\varepsilon_{\mathrm{Nd}}$ values between -4.8 and -4.5 . 
Table 2. C-H-O isotope composition of mineralisation at the Jianchaling Au deposit (modified from [21]).

\begin{tabular}{|c|c|c|c|c|c|c|c|c|c|c|}
\hline Sample No. & Sample & Vein Set & Mineral & $\delta^{18} \mathrm{O}_{\text {mineral }}(\%)$ & Average $\delta^{18} \mathrm{O}_{\text {Water }}(\%)$ & $\delta D_{\text {Water }}(\%)$ & $\delta^{13} C_{\text {V-PDB }}(\%)$ & Average $\delta^{13} \mathrm{C}_{\mathrm{CO} 2}(\%)$ & $T^{\#}\left({ }^{\circ} \mathrm{C}\right)$ & Reference \\
\hline MS-57 & Au mineralisation & 1 & quartz & 17.3 & $10.8(8.3$ to 12.1$)$ & -72 & & & 310 (250 to 355$)$ & [53] \\
\hline MS-79 & Au mineralisation & 1 & quartz & 17.0 & 10.5 (8.0 to 11.8 ) & -85 & & & 310 (250 to 355$)$ & [53] \\
\hline MS-52 & Au mineralisation & 1 & quartz & 16.6 & $10.1(7.6$ to 11.4$)$ & -79 & & & 310 (250 to 355$)$ & [53] \\
\hline MS-77 & Au mineralisation & 1 & quartz & 14.0 & 7.5 (5.0 to 8.8 ) & -70 & & & 310 (250 to 355$)$ & [53] \\
\hline $800-53-2$ & Au mineralisation & 1 & dolomite & 23.4 & 17.7 (15.7 to 18.8$)$ & & -0.3 & 1.7 (0.7 to 2.2$)$ & 310 (250 to 355$)$ & [53] \\
\hline $\mathrm{J} 6$ & Au mineralisation & 1 & dolomite & 18.9 & $13.2(11.2$ to 14.3$)$ & & 0.6 & $2.6(1.6$ to 3.1$)$ & 310 (250 to 355$)$ & [53] \\
\hline \multirow[t]{2}{*}{$860-52-7$} & Au mineralisation & 1 & dolomite & 19.1 & 13.4 (11.4 to 14.5$)$ & & -4.4 & $-2.4(-3.4$ to -1.9$)$ & 310 (250 to 355$)$ & [53] \\
\hline & Average & & & 18.0 & $11.9(9.6$ to 13.1$)$ & -77 & -1.4 & $0.6(-0.4$ to 1.1$)$ & & \\
\hline $913-32-6$ & Au mineralisation & 2 & calcite & 17.0 & 9.1 (7.2 to 11.2$)$ & & -2.9 & $-1.9(-3.0$ to -0.9$)$ & 240 (200 to 300$)$ & [21] \\
\hline $913-32-7$ & Au mineralisation & 2 & calcite & 16.5 & $8.6(6.7$ to 10.7$)$ & & -1.7 & $-0.7(-1.8$ to 0.3$)$ & 240 (200 to 300$)$ & [21] \\
\hline P633-61-I1 & Au mineralisation & 2 & calcite & 19.0 & 11.1 (9.2 to 13.2$)$ & & -0.6 & $0.4(-0.7$ to 1.4$)$ & 240 (200 to 300$)$ & [41] \\
\hline J163 & Au mineralisation & 2 & dolomite & 16.4 & $8.3(6.4$ to 10.4$)$ & & -1.6 & $-0.8(-1.8$ to 0.3$)$ & 240 (200 to 300$)$ & [41] \\
\hline $\mathrm{J} 170$ & Au mineralisation & 2 & dolomite & 13.8 & $5.7(3.8$ to 7.8$)$ & & -0.4 & $0.4(-0.6$ to 1.5$)$ & 240 (200 to 300$)$ & [41] \\
\hline $\mathrm{J} 180$ & Au mineralisation & 2 & dolomite & 14.6 & 6.5 (4.6 to 8.6$)$ & & -1.2 & $-0.4(-1.4$ to 0.7$)$ & 240 (200 to 300$)$ & [41] \\
\hline \multirow[t]{2}{*}{$\mathrm{J} 14$} & Au mineralisation & 2 & ferrocalcite & 17.5 & 9.6 (7.7 to 11.7$)$ & & -1.1 & $-0.1(-1.2$ to 0.9$)$ & 240 (200 to 300$)$ & [41] \\
\hline & Average & & & 16.4 & 8.4 (6.5 to 10.5$)$ & & -1.4 & $-0.4(-1.5$ to 0.6$)$ & & \\
\hline 913-1 & Au mineralisation & 3 & dolomite & 13.8 & $3.2(0.4$ to 4.8$)$ & & 1.6 & $1.0(-0.5$ to 1.9$)$ & 190 (150 to 220$)$ & [21] \\
\hline 913-2 & Au mineralisation & 3 & dolomite & 11.9 & $1.3(-1.5$ to 2.9$)$ & & 2.2 & $1.6(0.1$ to 2.5$)$ & $190(150$ to 220$)$ & [21] \\
\hline $960-38-1$ & Au mineralisation & 3 & dolomite & 13.1 & $2.5(-0.3$ to 4.1$)$ & & 0.6 & $0(-1.5$ to 0.9$)$ & 190 (150 to 220$)$ & [21] \\
\hline $860-51-5$ & Au mineralisation & 3 & dolomite & 14.8 & $4.2(1.4$ to 5.8$)$ & & -4.4 & $-5.0(-6.5$ to -4.1$)$ & 190 (150 to 220$)$ & [21] \\
\hline $860-51-6$ & Au mineralisation & 3 & dolomite & 14.9 & $4.3(1.5$ to 5.9$)$ & & -4.1 & $-4.7(-6.2$ to -3.8$)$ & $190(150$ to 220$)$ & [21] \\
\hline \multirow[t]{2}{*}{ MS-69 } & Au mineralisation & 3 & quartz & 16.3 & $3.9(0.8$ to 5.8$)$ & -81 & & & $190(150$ to 220$)$ & [41] \\
\hline & Average & 3 & & 14.1 & $3.2(0.4$ to 4.9$)$ & & -0.8 & $-0.7(-2.9$ to -0.5$)$ & & \\
\hline P404-44B-2 & $\begin{array}{c}\text { Mineralized } \\
\text { dolostone }\end{array}$ & & dolomite & 22.9 & & & 0.0 & & & [41] \\
\hline P404-43B & Altered dolostone & & dolomite & 18.0 & & & 0.1 & & & [41] \\
\hline P406-Y-43w & Altered dolostone & & dolomite & 16.9 & & & 0.7 & & & [41] \\
\hline $\mathrm{J} 142$ & Dolostone & & dolomite & 22.5 & & & 2.3 & & & [41] \\
\hline J171 & Dolostone & & dolomite & 23.1 & & & 2.3 & & & [41] \\
\hline \multirow[t]{2}{*}{$\mathrm{J} 25$} & Dolostone & & dolomite & 22.3 & & & -0.4 & & & [41] \\
\hline & Average & & & 21.0 & & & 0.8 & & & [41] \\
\hline $\mathrm{J} 167$ & Serpentinite & & dolomite & 15.0 & & & -3.8 & & & [41] \\
\hline $\mathrm{J} 42-1$ & Listwanite & & magnesite & 14.3 & & & -2.7 & & & [41] \\
\hline $\mathrm{J} 59$ & Listwanite & & magnesite & 16.3 & & & -3 & & & [41] \\
\hline Y-Mg & Listwanite & & listwanite & 13.8 & & & -3.2 & & & [41] \\
\hline Y-Mg & Listwanite & & listwanite & 13.8 & & & -3.2 & & & [41] \\
\hline \multirow[t]{2}{*}{ G-Mg } & Listwanite & & listwanite & 13.4 & & & -2.2 & & & [41] \\
\hline & Average & & & 14.5 & & & -3.0 & & & \\
\hline
\end{tabular}

Notation: ${ }^{\#}$ the peak of homogenization temperatures (the range of homogenization temperatures) are from [39]. The $\delta^{18} \mathrm{O}_{\mathrm{wtaer}}$ values
and carbonate-water $[54,55]$. The $\delta^{13} \mathrm{C}$ in $\mathrm{CO}_{2}$ equilibrated with dolomite and calcite were calculated using the equations of $[56,57]$ 
Table 3. Sulfur isotope composition of sulfides and whole rocks in the Jianchaling area (modified from [21]).

\begin{tabular}{|c|c|c|c|c|c|}
\hline Sample & Mineral & $\delta^{34} S_{\mathrm{v}-\mathrm{CDT}}(\%)$ & Sample & Mineral & $\delta^{34} S_{\mathrm{v}-\mathrm{CDT}}(\%)$ \\
\hline hjy-1 (second vein set) ${ }^{\mathrm{c}}$ & Py & 11.3 & Carbonate (Duantouya Fm) ${ }^{a}$ & Py & 16.4 \\
\hline hjy-12 (second vein set) ${ }^{c}$ & Py & 10.0 & Carbonaceous shale (Duantouya Fm) ${ }^{a}$ & Py & 5.4 \\
\hline hjy-10 (second vein set) ${ }^{c}$ & Py & 10.8 & Carbonaceous shale (Duantouya Fm) ${ }^{a}$ & Py & 10.6 \\
\hline $\mathrm{J} 3$ (second vein set) $^{\mathrm{c}}$ & Po & 8.2 & Carbonate (Duantouya Fm) ${ }^{a}$ & Py & 12.5 \\
\hline $\mathrm{J} 3$ (second vein set) $^{\mathrm{c}}$ & Py & 8.7 & Hc-Py1 (serpentinite) ${ }^{a}$ & Cpy & 12.0 \\
\hline hjy-20 (second vein set) ${ }^{c}$ & Py & 13.2 & L-Py-Mt (listwanite) $^{\text {a }}$ & Po & 10.5 \\
\hline hjy-14 (second vein set) ${ }^{c}$ & Py & 14.3 & Ni-bearing ultramafics ${ }^{a}$ & Py & 9.3 \\
\hline hjy-8 (first vein set) ${ }^{c}$ & Py & 14.3 & Ni-bearing ultramafics ${ }^{a}$ & Py & 11.3 \\
\hline H-4 Shale (Duantouya Formation) ${ }^{c}$ & Whole rock & 16.6 & Ni-bearing ultramafics ${ }^{a}$ & Py & 13.2 \\
\hline 800-52-1 (listwanite) ${ }^{\mathrm{c}}$ & Whole rock & 6.1 & Ni-bearing ultramafics ${ }^{a}$ & Py & 10.9 \\
\hline 780-50-3 (serpentinite) ${ }^{c}$ & Whole rock & 8.6 & Ni-bearing ultramafics ${ }^{a}$ & Py & 11.1 \\
\hline 965-Y-45-46 II & Py & 8.5 & Ni-bearing ultramafics ${ }^{a}$ & Py & 9.5 \\
\hline PD503-Y-Py1-2 II & Py & 15.4 & Ni-bearing ultramafics ${ }^{a}$ & Py & 11.7 \\
\hline PD404-cm43-Py ${ }^{\mathrm{a}}$ & Py & 13.9 & Ni-bearing ultramafics ${ }^{a}$ & Py & 10.5 \\
\hline PD406-cm45N-Py ${ }^{\text {a }}$ & Py & 14.2 & Ni-bearing ultramafics ${ }^{a}$ & Py & 12.3 \\
\hline PD404-cm45S-Py a & Py & 18.5 & Ni-bearing ultramafics ${ }^{a}$ & Py & 14.1 \\
\hline PD503-Ym-Py3 ${ }^{\mathrm{a}}$ & Py & 17.3 & Ni-bearing ultramafics ${ }^{\text {a }}$ & Py & 12.1 \\
\hline PD404-cm43B-Py1 ${ }^{\text {a }}$ & Py & 12.8 & Ni-bearing ultramafics ${ }^{a}$ & Py & 11.7 \\
\hline $965-47 \mathrm{E}-\mathrm{Ym}^{\mathrm{a}}$ & Rea, Orp & 10.9 & Ni-bearing ultramafics ${ }^{a}$ & Py & 11.7 \\
\hline PD383-cm58-H1 a & Rea, Orp & 10.5 & Ni-bearing ultramafics ${ }^{a}$ & Py & 11.4 \\
\hline PD633-cm43B-Py1 ${ }^{\text {a }}$ & Py & 12.6 & Ni-bearing ultramafics ${ }^{a}$ & Py & 10.0 \\
\hline G-E-1 ${ }^{\text {a }}$ & Py & 17.0 & Ni-bearing ultramafics ${ }^{a}$ & Py & 10.9 \\
\hline PD383-Y-Py1 ${ }^{a}$ & Py & 12.6 & Ni-bearing ultramafics ${ }^{a}$ & Py & 6.1 \\
\hline PD633-Ym-59 a & Py & 12.0 & Ni-bearing ultramafics ${ }^{a}$ & Py & 12.9 \\
\hline 97-ZHE-H1 ${ }^{\text {a }}$ & Py & 11.2 & Ni-bearing ultramafics ${ }^{a}$ & Po & 7.8 \\
\hline Au mineralisation & Py & 6.3 & Ni-bearing ultramafics ${ }^{a}$ & Po & 10.6 \\
\hline Au mineralisation & Py & 15.3 & Ni-bearing ultramafics ${ }^{a}$ & Po & 9.1 \\
\hline
\end{tabular}


Table 3. Cont

\begin{tabular}{|c|c|c|c|c|c|}
\hline Sample & Mineral & $\delta^{34} S_{\mathrm{v}-\mathrm{CDT}}(\%)$ & Sample & Mineral & $\delta^{34} S_{\text {v-CDT }}(\%)$ \\
\hline Sm-P (albite porphyry) ${ }^{a}$ & $\mathrm{Py}$ & 11.0 & Ni-bearing ultramafics ${ }^{a}$ & Po & 10.0 \\
\hline N124 (albite porphyry) ${ }^{a}$ & $\mathrm{Py}$ & 14.6 & Ni-bearing ultramafics ${ }^{a}$ & $\mathrm{Po}$ & 12.5 \\
\hline L-Py (albite porphyry) ${ }^{a}$ & Py & 12.9 & Ni-bearing ultramafics ${ }^{a}$ & Po & 10.7 \\
\hline Porphyritic granite ${ }^{a}$ & $\mathrm{Py}$ & 11.8 & Ni-bearing ultramafics ${ }^{a}$ & Po & 12.3 \\
\hline Porphyritic granite ${ }^{a}$ & Py & 12.8 & Ni-bearing ultramafics ${ }^{a}$ & Po & 11.4 \\
\hline Porphyritic granite $^{a}$ & Py & 17.5 & Ni-bearing ultramafics ${ }^{\text {a }}$ & Po & 10.9 \\
\hline Porphyritic granite ${ }^{a}$ & Po & 11.6 & Ni-bearing ultramafics ${ }^{a}$ & Po & 11.4 \\
\hline Porphyritic granite ${ }^{a}$ & Po & 13.7 & Ni-bearing ultramafics a & Po & 11.8 \\
\hline Porphyritic granite ${ }^{a}$ & Py & 9.0 & Ni-bearing ultramafics ${ }^{a}$ & $\mathrm{Po}$ & 11.1 \\
\hline Porphyritic granite ${ }^{a}$ & $\mathrm{Py}$ & 15.2 & Ni-bearing ultramafics ${ }^{a}$ & Po & 8.2 \\
\hline Porphyritic granite ${ }^{a}$ & Py & 15.2 & Ni-bearing ultramafics ${ }^{a}$ & Po & 10.5 \\
\hline Porphyritic granite $^{\text {a }}$ & Py & 15.2 & Ni-bearing ultramafics ${ }^{a}$ & Po & 11.6 \\
\hline Porphyritic granite $^{\text {a }}$ & Py & 8.3 & Ni-bearing ultramafics ${ }^{a}$ & Po & 10.2 \\
\hline Porphyritic granite $^{\text {a }}$ & Py & 10.6 & Ni-bearing ultramafics ${ }^{a}$ & Py & 10.3 \\
\hline Porphyritic granite $^{a}$ & Py & 9.5 & Ni-bearing ultramafics ${ }^{a}$ & Py & 12.1 \\
\hline Porphyritic granite ${ }^{a}$ & Py & 9.9 & Ni-bearing ultramafics a & Py & 9.9 \\
\hline Carbonate (Duantouya Fm) ${ }^{a}$ & Py & 15.0 & Ni-bearing ultramafics ${ }^{a}$ & Py & 11.4 \\
\hline Carbonate (Duantouya Fm) ${ }^{a}$ & Py & 18.5 & Ni-bearing ultramafics ${ }^{a}$ & Py & 11.5 \\
\hline Carbonate (Duantouya Fm) ${ }^{a}$ & Po & 18.6 & Ni-bearing ultramafics ${ }^{a}$ & Py & 13.3 \\
\hline Carbonate (Duantouya Fm) ${ }^{a}$ & Po & 16.5 & Ni-bearing ultramafics ${ }^{a}$ & Py & 12.3 \\
\hline Carbonate (Duantouya Fm) ${ }^{a}$ & Py & 10.3 & Porphyritic granite ${ }^{b}$ & Py & 10.0 \\
\hline
\end{tabular}

Superscripts: ${ }^{\mathrm{a}}[41] ;{ }^{\mathrm{b}}[50] ;{ }^{\mathrm{c}}$ [21]. Abbreviations: Cyp = chalcopyrite; Orp = orpiment; Py = pyrite, Po = pyrrhotite; Rea $=$ realgar. 
Table 4. Lead isotope composition of ore sulfide and wallrocks at the Jianchaling deposit and in the Bikou Group.

\begin{tabular}{|c|c|c|c|c|c|c|c|c|c|c|c|}
\hline Sample No. & Sample & $\mathrm{Pb}$ & Th & $\mathbf{U}$ & ${ }^{208} \mathrm{~Pb} /{ }^{204} \mathrm{~Pb}$ & ${ }^{207} \mathrm{~Pb} /{ }^{204} \mathrm{~Pb}$ & ${ }^{206} \mathrm{~Pb} /{ }^{204} \mathrm{~Pb}$ & $\left({ }^{208} \mathrm{~Pb} /{ }^{204} \mathrm{~Pb}\right)_{\mathrm{i}}$ & $\left({ }^{207} \mathrm{~Pb} /{ }^{204} \mathrm{~Pb}\right)_{\mathrm{i}}$ & $\left({ }^{206} \mathrm{~Pb} /{ }^{204} \mathrm{~Pb}\right)_{\mathrm{i}}$ & Reference \\
\hline \multicolumn{12}{|c|}{ Au mineralised-rocks } \\
\hline hjy-1 & pyrite & 905.000 & 0.333 & 0.216 & 38.757 & 15.704 & 18.477 & 38.757 & 15.704 & 18.477 & This study \\
\hline hjy-12 & pyrite & 170.000 & 0.070 & 0.158 & 37.996 & 15.539 & 18.126 & 37.996 & 15.539 & 18.124 & This study \\
\hline hjy-10 & pyrite & 50.000 & 0.035 & 0.539 & 37.088 & 15.550 & 17.600 & 37.088 & 15.549 & 17.579 & This study \\
\hline hjy-20 & pyrite & 60.900 & 0.266 & 0.047 & 37.180 & 15.566 & 17.522 & 37.177 & 15.566 & 17.521 & This study \\
\hline hjy-14 & pyrite & 53.800 & 0.041 & 0.193 & 37.135 & 15.577 & 17.683 & 37.135 & 15.577 & 17.676 & This study \\
\hline hjy-8 & pyrite & 352.000 & 0.019 & 0.068 & 37.275 & 15.549 & 17.811 & 37.275 & 15.549 & 17.811 & This study \\
\hline \multirow[t]{4}{*}{$\mathrm{J} 3$} & pyrite & 395.000 & 0.064 & 0.226 & 38.547 & 15.611 & 18.381 & 38.547 & 15.611 & 18.380 & This study \\
\hline & pyrrhotite & & & & 37.493 & 15.579 & 17.931 & & & & [41] \\
\hline & pyrite & & & & 37.055 & 15.553 & 17.863 & & & & [41] \\
\hline & pyrite & & & & 36.927 & 15.530 & 17.257 & & & & [41] \\
\hline Average & & & & & 37.545 & 15.576 & 17.865 & 37.711 & 15.585 & 17.938 & \\
\hline \multicolumn{12}{|c|}{ Meta-ultramafic rocks } \\
\hline & listwanite & & & & 36.029 & 15.520 & 17.952 & & & & [41] \\
\hline & listwanite & & & & 38.591 & 15.521 & 18.599 & & & & [41] \\
\hline & listwanite & & & & 38.604 & 15.785 & 19.193 & & & & [41] \\
\hline \multirow[t]{4}{*}{$800-52-1$} & listwanite & 21.500 & 0.016 & 0.062 & 38.069 & 15.641 & 18.723 & 38.069 & 15.641 & 18.717 & This study \\
\hline & listwanite & & & & 38.150 & 15.597 & 18.260 & & & & {$[41]$} \\
\hline & antigorite & & & & 38.118 & 15.632 & 18.390 & & & & [41] \\
\hline & antigorite & & & & 38.489 & 15.616 & 18.633 & & & & [41] \\
\hline \multirow[t]{4}{*}{$780-50-3$} & serpentinite & 31.500 & 0.041 & 0.052 & 37.748 & 15.615 & 18.327 & 37.747 & 15.615 & 18.324 & This study \\
\hline & serpentinite & & & & 38.920 & 15.657 & 18.553 & & & & [41] \\
\hline & serpentinite & & & & 38.032 & 15.570 & 18.833 & & & & [41] \\
\hline & meta-ultramafic & & & & 38.533 & 15.536 & 18.281 & & & & [58] \\
\hline Average & & & & & 38.117 & 15.608 & 18.522 & 37.908 & 15.628 & 18.521 & \\
\hline
\end{tabular}


Table 4. Cont

\begin{tabular}{|c|c|c|c|c|c|c|c|c|c|c|c|}
\hline Sample No. & Sample & $\mathrm{Pb}$ & Th & $\mathbf{U}$ & ${ }^{208} \mathrm{~Pb} /{ }^{204} \mathrm{~Pb}$ & ${ }^{207} \mathrm{~Pb} /{ }^{204} \mathrm{~Pb}$ & ${ }^{206} \mathrm{~Pb} /{ }^{204} \mathrm{~Pb}$ & $\left({ }^{208} \mathrm{~Pb} /{ }^{204} \mathrm{~Pb}\right)_{\mathrm{i}}$ & $\left({ }^{207} \mathrm{~Pb} /{ }^{204} \mathrm{~Pb}\right)_{\mathrm{i}}$ & $\left({ }^{206} \mathrm{~Pb} / 204 \mathrm{~Pb}\right)_{\mathrm{i}}$ & Reference \\
\hline \multicolumn{12}{|c|}{ Yudongzi Fm } \\
\hline \multirow{2}{*}{\multicolumn{5}{|c|}{$\begin{array}{l}\text { Sericite-quartz-schist } \\
\text { leptynite }\end{array}$}} & 38.204 & 15.663 & 18.432 & & & & [41] \\
\hline & & & & & 39.161 & 15.481 & 17.863 & & & & [41] \\
\hline Average & & & & & 38.683 & 15.572 & 18.148 & & & & \\
\hline \multicolumn{12}{|c|}{ Porphyritic granite } \\
\hline & magnetite & & & & 40.359 & 15.805 & 21.881 & & & & [41] \\
\hline & pyrrhotite & & & & 39.265 & 15.771 & 20.922 & & & & [41] \\
\hline & pyrite & & & & 38.338 & 15.765 & 21.637 & & & & [41] \\
\hline Average & & & & & 39.321 & 15.780 & 21.480 & & & & \\
\hline \multicolumn{12}{|c|}{ Duantouya Fm } \\
\hline $\mathrm{H} 4$ & shale & 115.000 & 9.120 & 4.580 & 39.156 & 15.768 & 19.819 & 39.103 & 15.764 & 19.737 & This study \\
\hline $960-34-1$ & dolostone & 15.600 & 0.064 & 0.250 & 37.518 & 15.667 & 19.164 & 37.515 & 15.665 & 19.132 & This study \\
\hline $960-28-1$ & dolostone & 31.800 & 0.016 & 0.217 & 38.170 & 15.828 & 22.364 & 38.170 & 15.827 & 22.350 & This study \\
\hline & limestone & & & & 38.172 & 15.601 & 18.365 & & & & [41] \\
\hline & dolomite & & & & 38.783 & 15.560 & 18.931 & & & & [58] \\
\hline Average & & & & & 38.360 & 15.685 & 19.729 & 38.263 & 15.752 & 20.406 & \\
\hline \multicolumn{12}{|c|}{ Bikou Group } \\
\hline & spilite & & & & 37.621 & 15.491 & 17.661 & & & & [59] \\
\hline & phyllite & & & & 35.328 & 15.383 & 16.308 & & & & [59] \\
\hline & phyllite & & & & 36.951 & 15.447 & 17.076 & & & & [59] \\
\hline & quartz schist & & & & 37.866 & 15.486 & 17.677 & & & & [59] \\
\hline & phyllite & 3.2 & 1.3 & 0.4 & 38.128 & 15.471 & 17.644 & 37.869 & 15.459 & 17.399 & [59] \\
\hline & siltstone & 3.2 & 1.3 & 0.4 & 38.394 & 15.552 & 18.126 & 38.132 & 15.540 & 17.879 & [59] \\
\hline & phyllite & 3.2 & 1.3 & 0.4 & 38.064 & 15.553 & 18.016 & 37.803 & 15.541 & 17.770 & [59] \\
\hline & metadolerite & 11.8 & 7.58 & 1.99 & 40.069 & 15.928 & 18.763 & 39.639 & 15.911 & 18.417 & [60] \\
\hline & metadolerite & 1.14 & 0.34 & 0.12 & 39.157 & 15.833 & 18.017 & 38.962 & 15.822 & 17.806 & [60] \\
\hline & metadolerite & 1.39 & 0.26 & 0.09 & 38.945 & 15.814 & 17.910 & 38.823 & 15.808 & 17.781 & [60] \\
\hline Average & & & & & 38.052 & 15.596 & 17.720 & 38.538 & 15.680 & 17.842 & \\
\hline
\end{tabular}


Table 5. The Sr isotope ratios of sulfides and wallrocks at the Jianchaling Au deposit and in the Bikou Group.

\begin{tabular}{|c|c|c|c|c|c|c|c|c|c|}
\hline No. & Material & Vein Set & $\mathrm{Rb}(\mathrm{ppm})$ & Sr (ppm) & ${ }^{87} \mathrm{Rb} /{ }^{86} \mathrm{Sr}$ & ${ }^{87} \mathrm{Sr} /{ }^{86} \mathrm{Sr}$ & $2 \sigma$ & $\mathbf{I}_{\mathrm{sr}}$ & References \\
\hline \multicolumn{10}{|c|}{ Mineralised rock } \\
\hline hjy-1 & Pyrite & 2 & 1.15 & 2.48 & - & & & & This study \\
\hline hjy-10 & Pyrite & 2 & 0.561 & 2.92 & 0.556233 & 0.714179 & 0.001222 & 0.712613 & This study \\
\hline hjy-12 & Pyrite & 2 & 0.671 & 3.37 & 0.576465 & 0.714265 & 0.001260 & 0.712642 & This study \\
\hline hjy-14 & Pyrite & 2 & 0.885 & 6.72 & - & & & & This study \\
\hline hjy-20 & Pyrite & 2 & 0.405 & 2.87 & 0.408670 & 0.717080 & 0.000961 & 0.715929 & This study \\
\hline $\mathrm{J} 3$ & Pyrite & 2 & 0.531 & 1.58 & 0.972553 & 0.709447 & 0.002004 & 0.706709 & This study \\
\hline Average & $N=4$ & & & & & & & 0.711973 & \\
\hline PD404-43B & Altered dolostone & 2 & 36.20 & 89.60 & 13.164780 & 0.713400 & 0.002401 & 0.710107 & [41] \\
\hline PD404-44B-2 & Mineralised rock & 2 & 4.20 & 7.83 & 19.593624 & 0.718400 & 0.003180 & 0.714026 & [41] \\
\hline PD633-61-11 & Quartz & 2 & & & & 0.720900 & & & [41] \\
\hline PD633-Y-57 & Calcite & 3 & & & & 0.717300 & & & [41] \\
\hline Average & $N=2$ & & & & & & & 0.712066 & \\
\hline \multicolumn{10}{|c|}{ Wall rocks } \\
\hline $\mathrm{J}-15$ & Porphyritic granite & & 77.56 & 36.67 & 6.136344 & 0.735543 & 0.012494 & 0.718266 & [41] \\
\hline $\mathrm{J}-16$ & Porphyritic granite & & 83.39 & 48.79 & 4.959944 & 0.738159 & 0.010187 & 0.724194 & [41] \\
\hline $\mathrm{J}-18$ & Porphyritic granite & & 83.68 & 31.21 & 7.789501 & 0.749689 & 0.016065 & 0.727757 & [41] \\
\hline $\mathrm{J}-19$ & Porphyritic granite & & 64.78 & 30.82 & 6.100934 & 0.740388 & 0.012508 & 0.723210 & [41] \\
\hline$J-60$ & Porphyritic granite & & 58.00 & 220.70 & 0.760528 & 0.709768 & 0.001597 & 0.707627 & [41] \\
\hline $\mathrm{J}-66$ & Porphyritic granite & & 75.03 & 79.03 & 2.754493 & 0.735919 & 0.005704 & 0.728164 & [41] \\
\hline $\mathrm{J}-69$ & Porphyritic granite & & 86.94 & 35.30 & 7.143593 & 0.732922 & 0.014431 & 0.712809 & [41] \\
\hline L-Py1 & $\begin{array}{l}\text { Albite-rich } \\
\text { intrusive }\end{array}$ & & 21.20 & 58.80 & 1.044631 & 0.721900 & 0.002184 & 0.718959 & [41] \\
\hline Average & $N=8$ & & & & & & & 0.720123 & \\
\hline $\mathrm{J}-17$ & Meta-ultramafics & & 0.27 & 17.16 & 0.045573 & 0.718455 & 0.000498 & 0.718327 & [41] \\
\hline $\mathrm{J}-55$ & Meta-ultramafics & & 1.59 & 13.47 & 0.341431 & 0.704674 & 0.000827 & 0.703713 & [41] \\
\hline $\mathrm{J}-102$ & Meta-ultramafics & & 1.33 & 5.58 & 0.689301 & 0.702761 & 0.001446 & 0.700820 & [41] \\
\hline $\mathrm{J}-118$ & Meta-ultramafics & & 0.25 & 35.27 & 0.020501 & 0.703870 & 0.000472 & 0.703812 & [41] \\
\hline G-E-Mt & Meta-ultramafics & & 2.92 & 64.30 & 0.131452 & 0.712300 & 0.000549 & 0.711930 & [41] \\
\hline Hc-Fe-1(1) & Meta-ultramafics & & 0.46 & 6.59 & 0.202065 & 0.712800 & 0.000631 & 0.712231 & [41] \\
\hline Hg-N-1 & Meta-ultramafics & & 0.33 & 3.92 & 0.243695 & 0.712800 & 0.000688 & 0.712114 & [41] \\
\hline 97-Hw-1 & Meta-ultramafics & & 1.11 & 1.01 & 5.358452 & 0.729600 & 0.006523 & 0.720628 & [41] \\
\hline $\mathrm{J}-45$ & Meta-ultramafics & & 1.90 & 4.58 & 2.842641 & 0.725862 & 0.002510 & 0.722476 & [41] \\
\hline $\mathrm{J}-50$ & Meta-ultramafics & & 0.53 & 2.11 & 2.218363 & 0.717723 & 0.001553 & 0.715675 & [41] \\
\hline$J-42-3$ & Meta-ultramafics & & 0.28 & 0.69 & 4.384971 & 0.726283 & 0.002460 & 0.722971 & [41] \\
\hline $\mathrm{Y}-\mathrm{Mg}$ & Meta-ultramafics & & 0.33 & 3.66 & 1.153334 & 0.713400 & 0.000714 & 0.712665 & [41] \\
\hline G-Mg & Meta-ultramafics & & 0.29 & 2.57 & 1.666748 & 0.713400 & 0.000817 & 0.712480 & [41] \\
\hline $780-50-3$ & Meta-ultramafics & & 1.57 & 2.97 & 1.531251 & 0.719517 & 0.003140 & 0.715206 & This study \\
\hline $800-52-1$ & Meta-ultramafics & & 0.10 & 0.80 & 0.376258 & 0.711025 & 0.000895 & 0.709965 & This study \\
\hline Average & $N=15$ & & & & & & & 0.713001 & \\
\hline
\end{tabular}


Table 5. Cont

\begin{tabular}{|c|c|c|c|c|c|c|c|c|c|}
\hline No. & Material & Vein Set & $\mathrm{Rb}(\mathrm{ppm})$ & Sr (ppm) & ${ }^{87} \mathrm{Rb} /{ }^{86} \mathrm{Sr}$ & ${ }^{87} \mathrm{Sr} /{ }^{86} \mathrm{Sr}$ & $2 \sigma$ & $\mathrm{I}_{\mathrm{sr}}$ & References \\
\hline PX406-Y-43W & Duantouya Fm & & 1.69 & 108.00 & 0.447747 & 0.719000 & 0.000499 & 0.718872 & [41] \\
\hline G-E-1 & Duantouya Fm & & 8.89 & 101.00 & 2.691745 & 0.723300 & 0.000720 & 0.722582 & [41] \\
\hline $\mathrm{H} 4$ & Duantouya Fm & & 105.00 & 57.20 & 5.312134 & 0.734027 & 0.010856 & 0.719034 & This study \\
\hline $960-28-1$ & Duantouya Fm & & 0.03 & 17.90 & 0.004370 & 0.720582 & 0.000493 & 0.720570 & This study \\
\hline Average & $N=4$ & & & & & & & 0.720265 & \\
\hline 2000224 & Bikou Group & & 19.55 & 594.59 & 1.463281 & 0.704471 & 0.000507 & 0.704203 & [61] \\
\hline 2000225 & Bikou Group & & 5.33 & 118.58 & 2.088492 & 0.704899 & 0.000538 & 0.704533 & [61] \\
\hline 2000226 & Bikou Group & & 3.95 & 41.58 & 4.601666 & 0.703211 & 0.000720 & 0.702438 & [61] \\
\hline 2000228 & Bikou Group & & 3.87 & 18.15 & 10.756975 & 0.703534 & 0.001312 & 0.701797 & [61] \\
\hline 2000230 & Bikou Group & & 3.73 & 39.70 & 4.931685 & 0.702650 & 0.000715 & 0.701884 & [61] \\
\hline 2000231 & Bikou Group & & 23.01 & 243.96 & 5.126925 & 0.713325 & 0.000732 & 0.712556 & [61] \\
\hline BK1 & Bikou Group & & 23.40 & 563.00 & 2.342130 & 0.710419 & 0.000536 & 0.710080 & [60] \\
\hline BK2 & Bikou Group & & 0.21 & 111.00 & 0.110386 & 0.707353 & 0.000475 & 0.707338 & [60] \\
\hline BK3 & Bikou Group & & 0.13 & 143.00 & 0.054847 & 0.706668 & 0.000474 & 0.706661 & {$[60]$} \\
\hline Average & $N=9$ & & & & & & & 0.705721 & \\
\hline
\end{tabular}

Table 6. The Nd isotope ratios of sulfides and wallrocks at the Jianchaling deposit.

\begin{tabular}{|c|c|c|c|c|c|c|c|c|c|}
\hline No. & Sample & Stage & $\mathrm{Sm}$ & Nd & ${ }^{143} \mathrm{Nd} /{ }^{144} \mathrm{Nd}$ & ${ }^{147} \mathrm{Sm} /{ }^{144} \mathrm{Nd}$ & $\left({ }^{143} \mathrm{Nd} /{ }^{144} \mathrm{Nd}\right) \mathrm{i}$ & $\mathrm{f}_{\mathrm{Sm} / \mathrm{Nd}}$ & $\varepsilon_{N d}(198)$ \\
\hline \multicolumn{10}{|c|}{ Sulfides } \\
\hline hjy-1 & Pyrite & 2 & 0.052 & 0.167 & 0.512426 & 0.1883 & 0.512182 & -0.04 & -3.9 \\
\hline hjy-10 & Pyrite & 2 & & 0.018 & & & & & \\
\hline hjy-12 & Pyrite & 2 & 0.085 & 0.747 & 0.513300 & 0.0688 & 0.512453 & -0.65 & 1.4 \\
\hline hjy-14 & Pyrite & 2 & 0.012 & 0.041 & 0.511941 & 0.1769 & 0.511712 & -0.10 & -13.1 \\
\hline hjy-20 & Pyrite & 2 & 0.06 & 0.425 & 0.511500 & 0.0853 & 0.511389 & -0.57 & -19.4 \\
\hline $\mathrm{J} 3$ & Pyrite & 2 & 0.026 & 0.194 & 0.511481 & 0.0810 & 0.511376 & -0.59 & -19.6 \\
\hline Average & $N=5$ & & & & & & 0.511823 & & -10.9 \\
\hline \multicolumn{10}{|c|}{ Wallrocks } \\
\hline $\mathrm{H} 4$ & Duantouya Fm & & 3.79 & 16 & 0.512051 & 0.1432 & 0.511865 & -0.27 & -10.1 \\
\hline 960-34-1 & Duantouya Fm & & 0.216 & 1.29 & 0.512012 & 0.1012 & 0.511881 & -0.49 & -9.8 \\
\hline $960-28-1$ & Duantouya Fm & & 0.039 & 0.129 & 0.512350 & 0.1828 & 0.512114 & -0.07 & -5.3 \\
\hline Average & $N=3$ & & & & & & 0.511953 & -0.28 & -8.4 \\
\hline $780-50-3$ & $\begin{array}{l}\text { Meta-ultramafic } \\
\text { rock }\end{array}$ & & 0.058 & 0.242 & 0.512325 & 0.1449 & 0.512137 & -0.26 & -4.8 \\
\hline $800-52-1$ & $\begin{array}{l}\text { Meta-ultramafic } \\
\text { rock }\end{array}$ & & 0.004 & 0.013 & 0.512393 & 0.1860 & 0.512152 & -0.05 & -4.5 \\
\hline Average & $N=2$ & & & & & & 0.512144 & -0.16 & -4.7 \\
\hline
\end{tabular}




\section{Discussion}

\subsection{Sources of Metals and Mineralising Fluids}

\subsubsection{Oxygen and Hydrogen Stable Isotopes}

The oxygen and hydrogen stable isotopes are often used to indicate the source of mineralised hydrothermal fluids [62]. However, the overlap in the metamorphic and magmatic fields in $\delta \mathrm{D}$ versus $\delta^{18} \mathrm{O}$ diagrams creates uncertainty in deciphering the genesis of mineralisation [63]. This is the case for the mineralising fluid at Jianchaling, which has been interpreted as being magmatic [64,65], meteoric [53], mixed metamorphic and magmatic [42,43,51], and primarily metamorphic [39]. This places a significant doubt on the usefulness of the $\delta \mathrm{D}$ and $\delta^{18} \mathrm{O}$ values in directly pinpointing the origin of the mineralising fluid at Jianchaling.

Using fluid-inclusion homogenisation temperatures and detailed paragenetic studies by Yue [39] (Table 2), the $\delta^{18} \mathrm{O}_{\text {water }}$ values were calculated using the quartz-water equation by [54] and carbonate-water equation by [55]. The calculated quartz-carbonate $\delta^{18} \mathrm{O}$ values for the hydrothermal fluid associated with the first vein set range from 7.5 to $17.7 \%$, the mineralised second vein set have $\delta^{18} \mathrm{O}$ values between 5.7 and $11.1 \%$, and the third vein set range from 1.3 to $4.3 \%$ (Table 2). Using this data, the $\delta^{18} \mathrm{O}$ and $\delta \mathrm{D}$ values for first vein set plot in the magmatic field and close to the metamorphic field (Figure 6). However, assuming that magmatic fluids are generated above the lowest eutectic point at temperatures above $573{ }^{\circ} \mathrm{C}$ [66], continuous cooling and water-rock reactions result in reduction

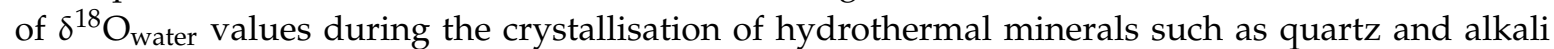
feldspar [66]. Furthermore, magmatic fluids have average $\delta^{18} \mathrm{O}_{\text {water values of } 18.8 \% \text { at temperatures }}$ of $355^{\circ} \mathrm{C}$ [39], which is the average homogenization temperature of fluid inclusions from first vein

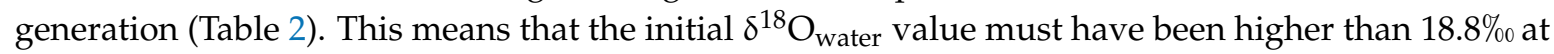
temperatures above $355^{\circ} \mathrm{C}$, which is higher than the $5.7-11.1 \%$ range for the mineralised second vein set. In addition, the Late Triassic to Early Jurassic plutons in the southern part of the Qinling Orogen commonly have $\delta^{18} \mathrm{O}$ values of $<18.8 \%$. The high $\delta^{18} \mathrm{O}$ values for the early hydrothermal fluids are consistent with a major contribution from metamorphic sources (rather than a minor contribution as suggested by Figure 6). This interpretation is supported by the low salinity and high $\mathrm{CO}_{2}$ content of fluid inclusions in the first vein set $[7,39]$. Furthermore, the $\delta^{18} \mathrm{O}$ values for quartz in granite porphyry and aplite in the area range between 11.3 and 14.5\% [67]. This indicates that the $\delta^{18} \mathrm{O}_{\text {water value of }}$ $10.0 \%$ calculated at a temperature of $\sim 573{ }^{\circ} \mathrm{C}$ for the first vein set cannot exceed the $\delta^{18} \mathrm{O}_{\text {water }}$ value of $13.2 \%$ for the second vein set that crystallised at $\sim 300{ }^{\circ} \mathrm{C}$ (as determined from the fluid inclusion homogenisation temperatures). In addition, the regional metamorphism in the Jianchaling area is at the greenschist facies [68], further supporting a $\sim 300^{\circ} \mathrm{C}$ temperature for the crystallisation of the mineralised second vein set.

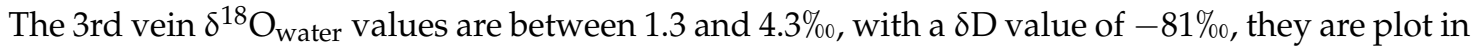
the meteoric field in Figures 6 and 7 . The $\delta 18$ Owater values of carbonate-quartz from the mineralised vein set vary between 5.7 and $11.1 \%$, which are values between those for the first and third vein sets.

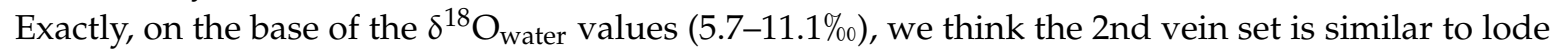
gold deposits and add the possible range of the 2nd vein set in Figure 6 that based on the feature of many lode gold deposits worldwide. This is indicative of the mixing of metamorphic and meteoric fluids during mineralisation (Figure 6).

The $\delta^{18} \mathrm{O}$ values for quartz from lode-gold deposits around the world are higher than $10 \%$ with the values for the mineralising fluids ranging between 5 and $25 \%$ [6]. The $\delta^{18} \mathrm{O}_{\text {water values of the gold }}$ deposits on the Jiaodong Peninsula of eastern China range from 4.9 to $10.9 \%$, with corresponding $\delta \mathrm{D}$ values of -78 to $-101 \%$ (Figure 6) $[69,70]$. The $\delta{ }^{18} \mathrm{O}$ and $\delta \mathrm{D}$ values for mineralising fluids at Jianchaling plot in the lode-gold deposit field (Figure 6; Table 2; [6]). Figure 6 also shows that the first vein set containing higher $\delta^{18} \mathrm{O}$ and $\delta \mathrm{D}$ values are sourced from metamorphic fluids. In contrast, 
the mineralised second vein set are similar lode gold deposits, and the fluids related to the third vein set have (again) a composition close to that of meteoric fluid.

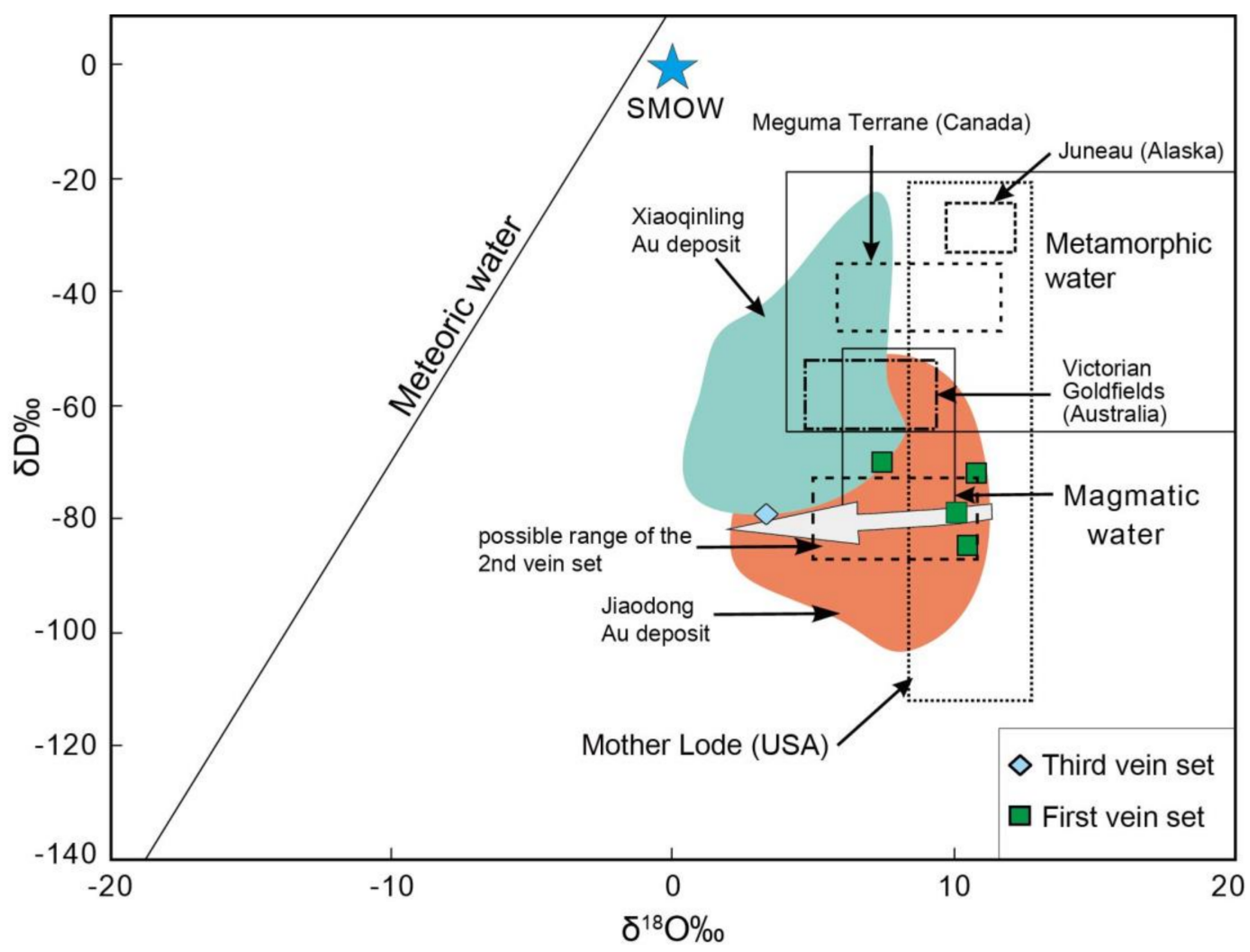

Figure 6. Stable isotope ( $\delta \mathrm{D}$ versus $\delta^{18} \mathrm{O}$ ) plot for mineralised fluids at the Jianchaling Au deposit [71]. Included are the fields for the Xiaoqinling and Jiaodong gold deposits [69]; Juneau gold belt, Mother Lode, Victorian Goldfields and Meguma Terrace Au deposits [6].

\subsubsection{Carbon and Oxygen Isotopes}

The $\delta^{13} \mathrm{C}_{\mathrm{CO} 2}$ values determined for quartz-carbonate from each of the vein sets are essentially the same, hovering around approximated zero (Figure 7). The corresponding $\delta^{18} \mathrm{O}_{\text {SMOW }}$ values show a significant trend with the first vein set being approximately $15 \%$, the mineralised second vein measuring around 9\%, and the third being around 3\% (Figure 7). The implication for this trend is that all of the vein sets have similar sources for carbon with a gradual decrease in available oxygen in the hydrothermal fluid with time. Another implication is that there was only one hydrothermal fluid that has changed in composition with time.

The $\delta^{13} \mathrm{C}_{\mathrm{CO} 2}$ values for the hydrothermal fluids related to the three vein sets are significantly higher than the values for organic matter (averaging -27\%) [62], the content of $\mathrm{CO}_{2}$ in the atmosphere ( -11 to $-7 \%$ [62]), freshwater carbonate ( -20 to $-9 \%$ [72]), magmatic rocks ( -30 to $-3 \%$ [72]), the continental crustal ( $-7 \%$ [73]), and the mantle ( -7 to $-5 \%$ [72]). However, marine carbonate (i.e., -3 to $2 \% 0 \delta^{13} \mathrm{C}_{\mathrm{CO} 2}$ [72]) and carbonate in the wall rock (i.e., $-0.4 \%$ to $2.3 \%_{0} \delta^{13} \mathrm{C}_{\mathrm{CO} 2}$; Table 2), are similar in $\delta^{13} \mathrm{C}_{\mathrm{CO} 2}$ composition. This indicates a local source for the hydrothermal fluids related to each of the vein sets. In addition, the close relationship between the ultramafic rocks and the vein sets shown in Figure 7 indicates that the ultramafic rocks are also local sources for the carbon and oxygen in the hydrothermal fluids. 


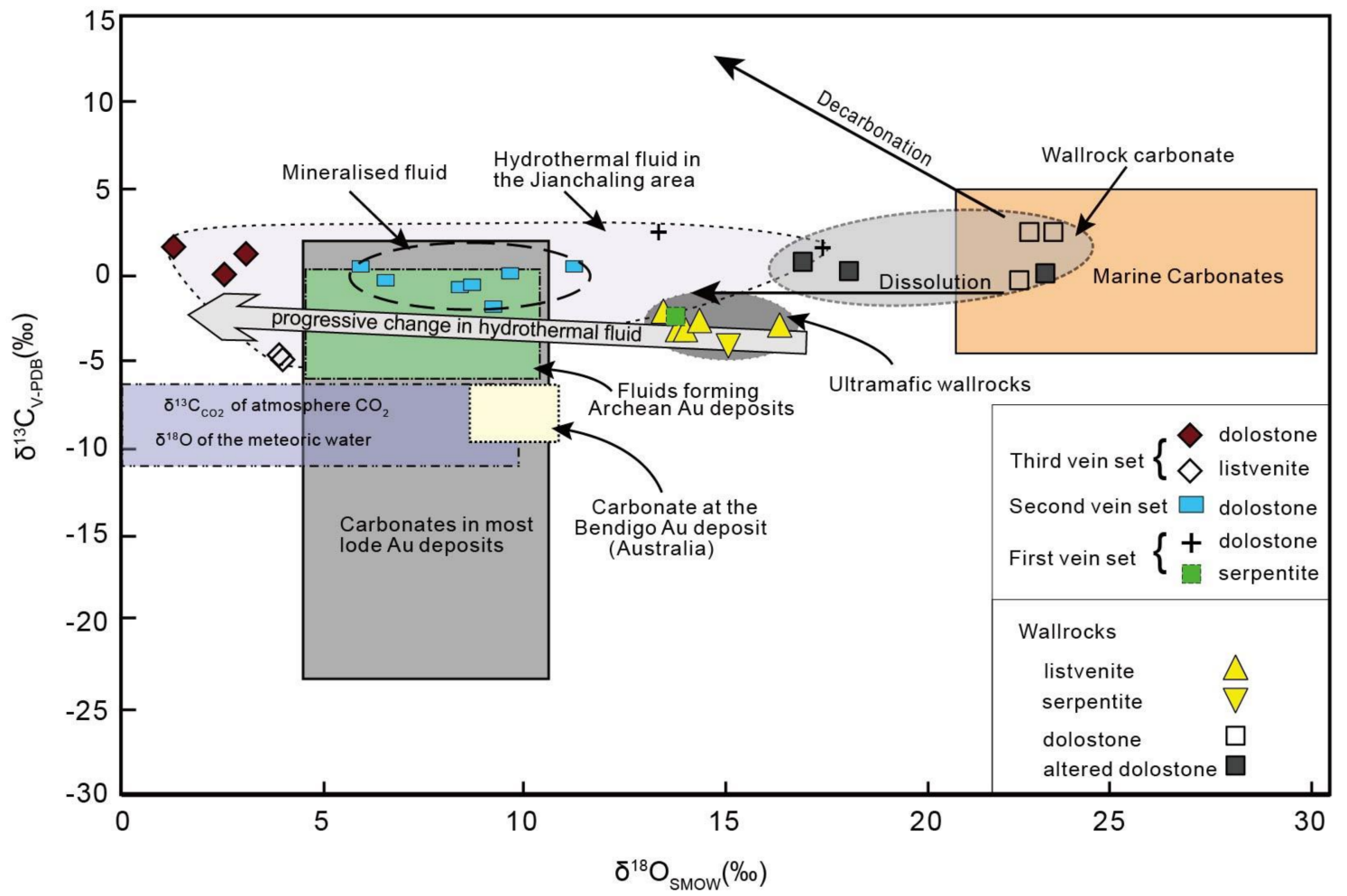

Figure 7. $\delta^{18} \mathrm{O}_{\mathrm{SMOW}}$ versus $\delta^{13} \mathrm{C}_{\mathrm{V}-\mathrm{PDB}}$ plot for the Jianchaling $\mathrm{Au}$ deposit [21]. Data sources: greenschist facies carbonate rocks after [74]; reduced C in sedimentary and metamorphic rocks after [75]; carbonates in most orogenic Au deposits after [76].

On the other hand, the $\delta^{13} \mathrm{C}_{\mathrm{CO} 2}$ and $\delta^{18} \mathrm{O}_{\mathrm{SMOW}}$ of the mineralised fluids overlap with those of the forming fluid of Archean Au deposits (Figure 7). $\delta^{18} \mathrm{O}_{\text {SMOW }}$ value of the third vein sets are similar with the meteoric water, indicating meteoric water mixed into the fluid system in late stage.

\subsubsection{Sulfur Isotopes}

The $\delta^{34} \mathrm{~S}$ signatures for various in the MLY and others from throughout the world are presented in Figure 8 . The figure shows that most of the sulfides in the MLY have $\delta^{34} \mathrm{~S}$ signatures greater than zero, are predominantly higher than those for orogenic Au deposits throughout the world, and higher than those for basaltic magmatic rocks, overlap the sulfide values in ultramafic rocks, and are coincide with the high end of sulfides in metamorphic rocks. In fact, the $\delta^{34} S$ signatures for the metamorphic rocks are the best fit, which includes the wallrocks at Jianchaling (Table 3). Such heavy $\delta^{34} S$ values for the sulfides at Jianchaling is consistent with derivation from sulfates that are characterised by heavy $\delta^{34} \mathrm{~S}$ values $[77,78]$. It is envisages that organic material derived by the metasedimentary rocks in the study area would have reacted with sulfate resulting in the crystallisation of sulfides [78,79]. Examples of such reducing agents are $\mathrm{CH}_{4}, \mathrm{C}_{2} \mathrm{H}_{6}, \mathrm{H}_{2} \mathrm{~S}$ and graphite, which have been detected in fluid inclusions using Laser Raman [39]. The conversion of sulfate to sulfide will proceed in the following chemical reaction:

$$
\mathrm{CH}_{4}+\mathrm{SO}_{4}{ }^{2-} \leftarrow \rightarrow \mathrm{H}_{2} \mathrm{~S}+\mathrm{H}_{2} \mathrm{O}+\mathrm{CO}_{3}{ }^{2-} \text { and } \mathrm{H}_{2} \mathrm{~S}+\mathrm{Fe}^{2+} \leftarrow \rightarrow \mathrm{FeS}+2 \mathrm{H}^{+}
$$

Therefore, the Neoproterozoic metamorphic rocks in the MLY are likely sources for sulfur in the Jianchaling area. 


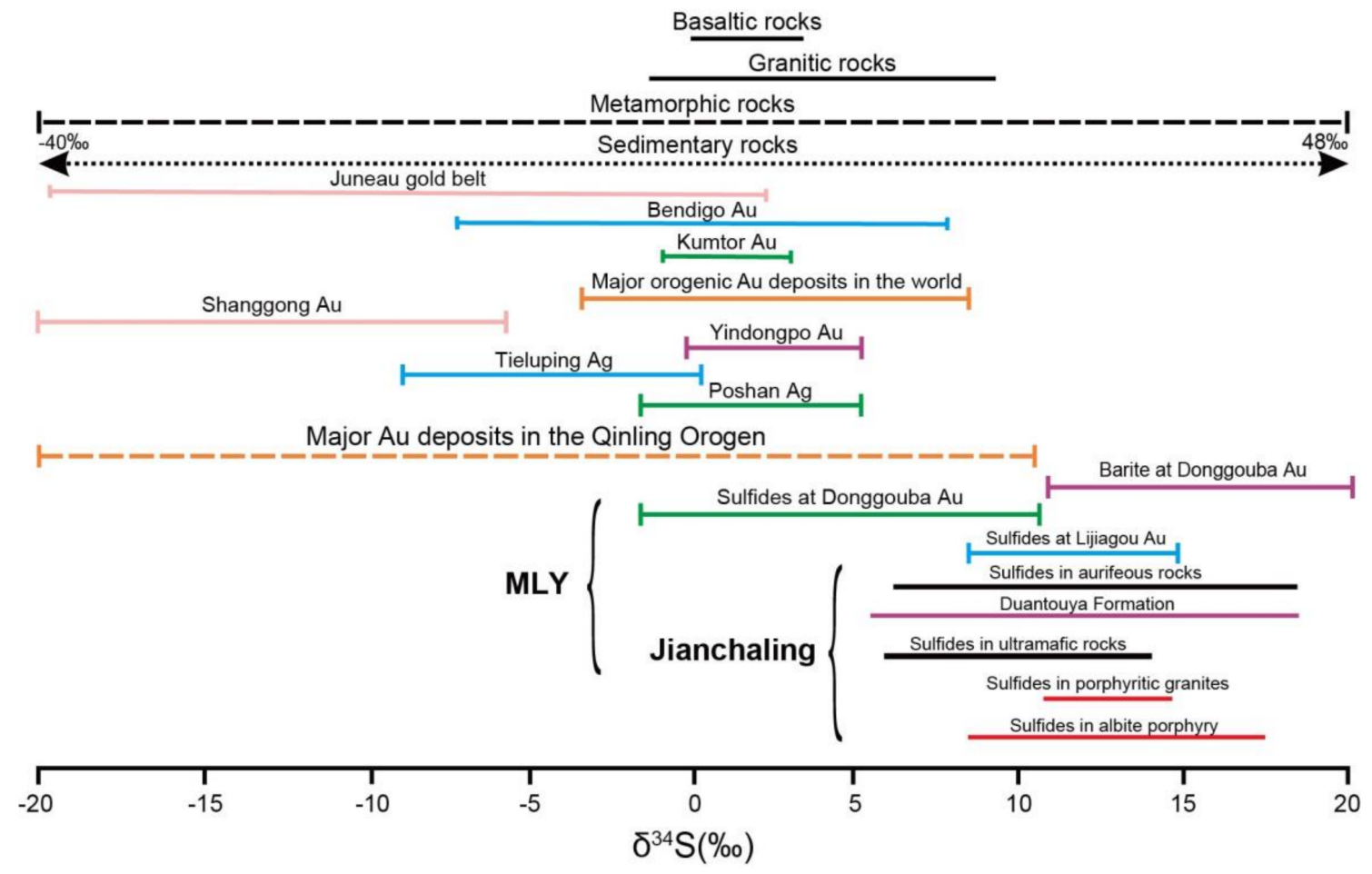

Figure 8. $\delta^{34} \mathrm{~S}$ values for hydrothermal sulfides from the Jianchaling Au deposit. Data sources: Basaltic rocks, Granitic rocks, Metamorphic rocks and Sedimentary rocks after [62,72,80]; Juneau gold belt, Bendigo Au, Kumtor Au and Major orogenic Au deposits in the world after [12,81]; Shanggong Au and Tieluping Ag after [44]; Yindongpo Au after [66]; Poshan Ag after [82]; Major Au deposits in the Qinling Orogen [24].

\subsubsection{Lead Isotopes}

The country rocks in the Jianchaling area have a wider range in $\mathrm{Pb}$ isotopic $\left({ }^{206} \mathrm{~Pb} /{ }^{204} \mathrm{~Pb}\right.$, ${ }^{207} \mathrm{~Pb} /{ }^{204} \mathrm{~Pb}$ and ${ }^{208} \mathrm{~Pb} /{ }^{204} \mathrm{~Pb}$ ) ratios than the sulfides in the Jianchaling mineralisation (Table 4). The range of the $\mathrm{Pb}$-isotopes is shown in Figure 9 that includes trends defining tectonic settings [83]. The range of $\mathrm{Pb}$-isotopes shown in Figure 9 correlates with defined tectonic setting of orogenic and upper crustal fields, even though there is a limited number of 10 data points. This is in agreement with the observations made in the discussion section above.

Furthermore, these $\mathrm{Pb}$ signatures of the sulfides from the mineralisation overlap those of the ultramafic rocks and the Bikou Group, indicating that the units are possible sources for the sulfides in the mineralisation. In contrast, the distinct incongruence of the sulfides from the mineralisation and the granitic rocks indicates that the granitic rocks are not the source for the sulfides in the mineralisation (Figure 9).

\subsubsection{Strontium and Neodymium Isotopes}

The $\mathrm{I}_{\mathrm{Sr}}(\mathrm{t})$ values of sulfide samples from the Jianchaling deposit range from 0.706709 to 0.715929 (Table 5; Figure 10a). These values are higher than the mean values (0.703 to 0.705) established for mantle-derived oceanic basalt (MORB, OIB, IAB) and continental basalt, and are suggestive of a crustal source $[84,85]$.

The $\mathrm{I}_{\mathrm{Sr}}(\mathrm{t})$ values for the sulfide samples from the deposit are also lower than those for the Duantouya Formation (Table 5), and the $\left({ }^{143} \mathrm{Nd} /{ }^{144} \mathrm{Nd}\right)$ i sulfide ratios for the mineralised second vein set overlap with those of the Duantouya Formation and the meta-ultramafic rocks (Table 6; Figure 10a,b). The $\varepsilon N d(t)$ and Isr $(t)$ of sulfide are indicative of a crustal source and dominated with mature Sr-Nd character (Tables 5 and 6; Figure 10a), and $\varepsilon N d$ (t) overlaps with meta-ultramafic field, 
indicated that the Duantouya may play an important role during the ore formation and there may exist minor ultramafic source been involved in the ore fluid.

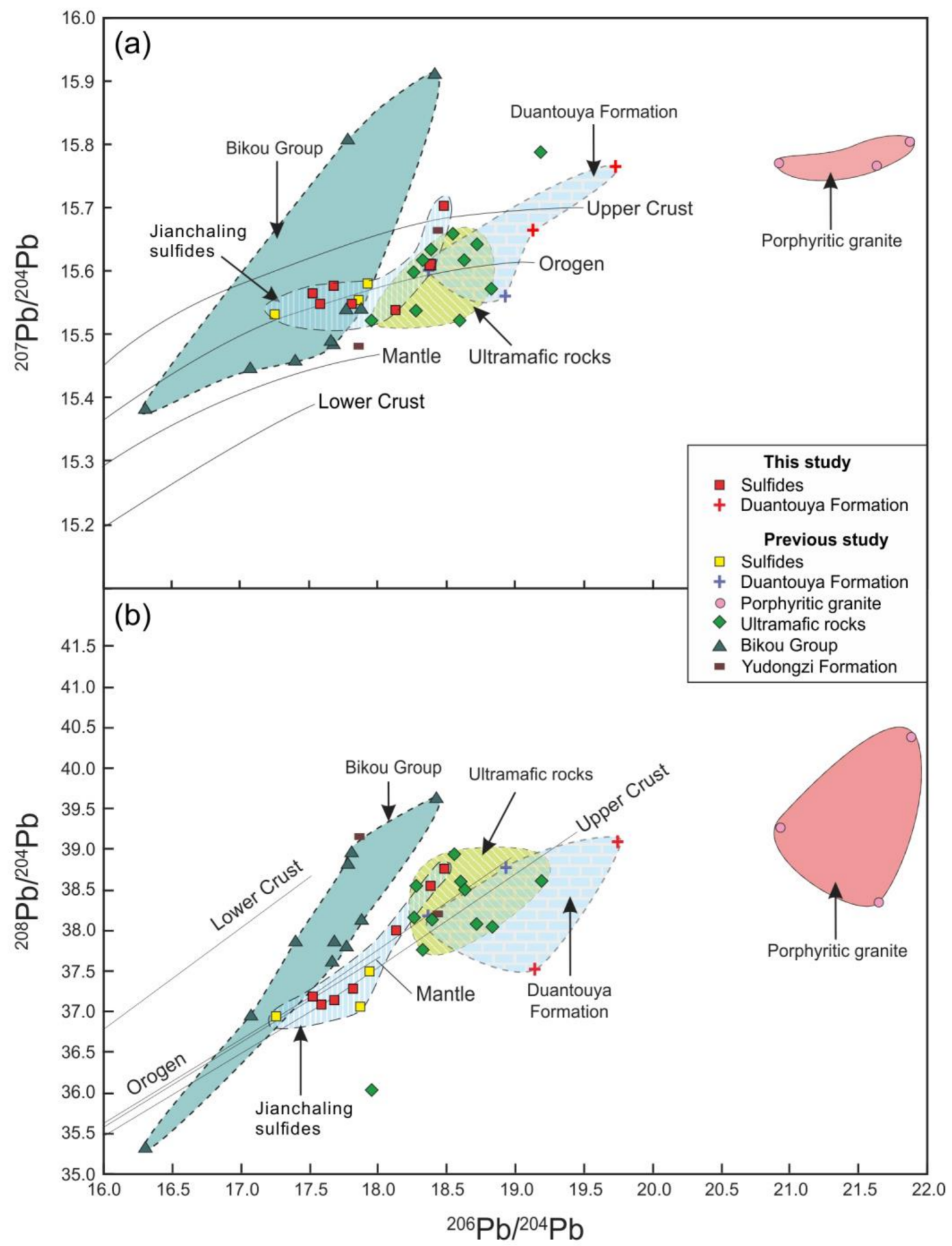

Figure 9. The plumbotectonic model for $\mathrm{Pb}$ isotopic systematics for the Jianchaling Au deposit. (a) ${ }^{206} \mathrm{~Pb} /{ }^{204} \mathrm{~Pb}$ versus ${ }^{208} \mathrm{~Pb} /{ }^{204} \mathrm{~Pb}$; (b) ${ }^{206} \mathrm{~Pb} /{ }^{204} \mathrm{~Pb}$ versus ${ }^{207} \mathrm{~Pb} /{ }^{204} \mathrm{~Pb}$. 
(a)

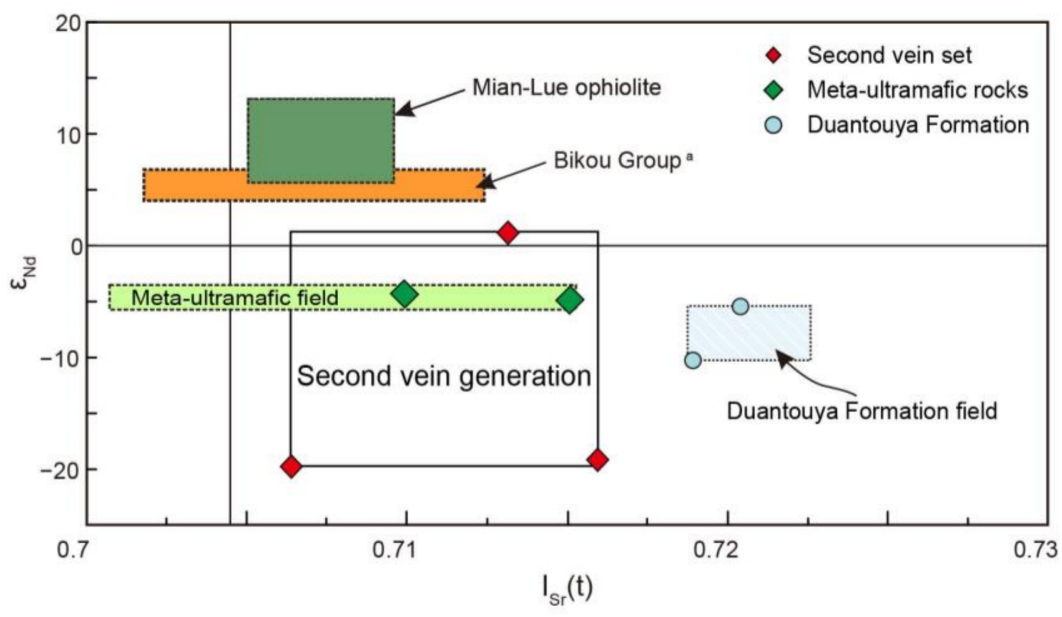

(b)

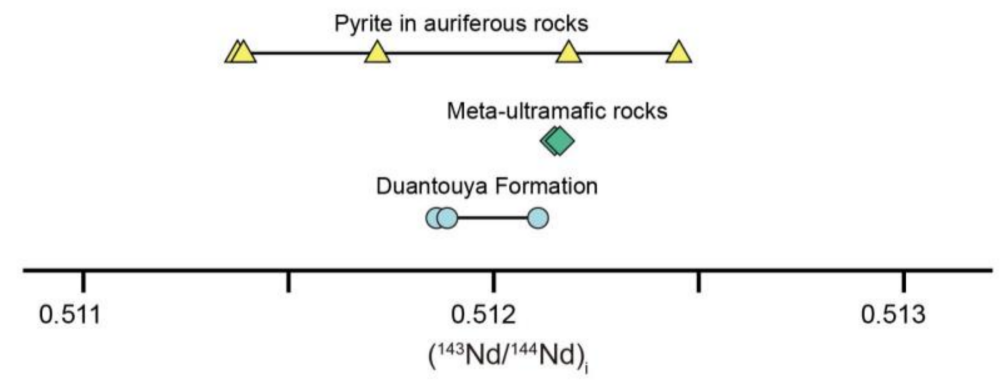

(c)

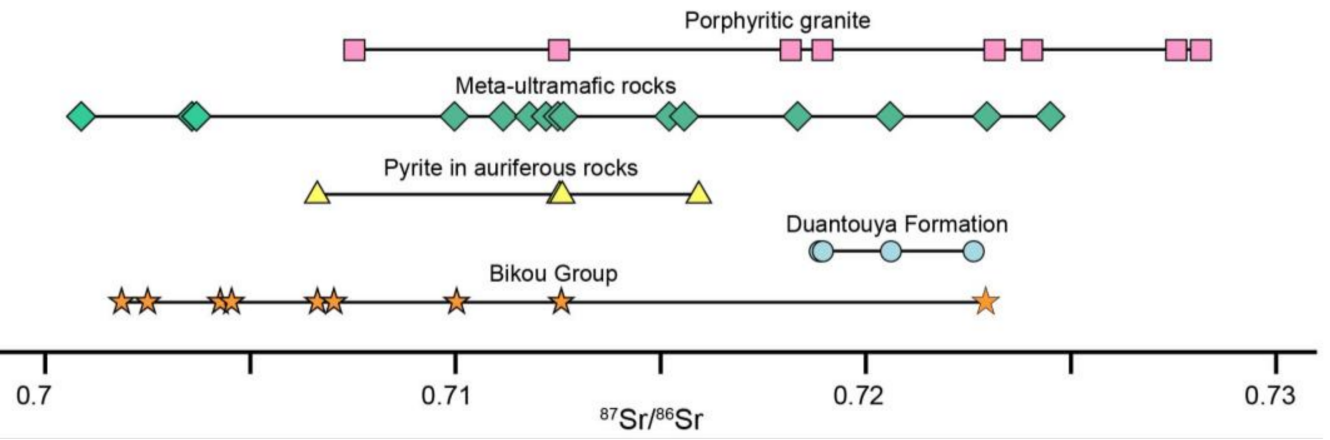

Figure 10. Isotope systematics for the Jianchaling Au deposit showing: (a) $\mathrm{I}_{\mathrm{Sr}}(\mathrm{t})$ versus $\varepsilon_{\mathrm{Nd}}(\mathrm{t})$ plot (data for the Bikou Group is from [86]); (b) $\left({ }^{143} \mathrm{Nd} /{ }^{144} \mathrm{Nd}\right) \mathrm{i}$ plot; and (c) ${ }^{87} \mathrm{Sr} /{ }^{86} \mathrm{Sr}$ plot. The range of the Mian-lue ophiolite is from [23]. T = 198 Ma.

The Bikou Group has $\mathrm{I}_{\mathrm{Sr}}(\mathrm{t})$ values that overlap with those from the Mian-Lue ophiolite, meta-ultramafic rocks, partly with the second vein set, and are lower than those from the Duantouya Formation (Figure 10a; Tables 5 and 6) $[60,61,86]$. Although the pyrite in audiferous rocks has $\mathrm{I}_{\mathrm{Sr}}(\mathrm{t})$ values that overlap with porphyritic granite partly (Figure 10c), it is unlikely to come from porphyritic granite, the evidence is mainly from lead isotopes, as mentioned above.

These relationships can be explained with a mixing model where the mineralised sulfide-bearing fluids interacted with lithological units (Figure 10). This interpretation is supported by the conclusion drawn from the $\mathrm{Pb}$ isotope data collected from the Huachanggou gold deposit in the northwestern part of MLY [25,59] (Figure 1c). 


\subsection{Mineralisation Type}

Various mineral-types have been proposed for the Jianchaling Au deposit. These include: (1) a magmatic hydrothermal source associated with Triassic magmatism [65]; (2) Carlin-like affiliations [8]; (3) orogenic affiliations [43]; and (4) sourced from structurally controlled altered rocks [40,53].

Fuchsite separated from the mineralised second vein set yield a well-defined ${ }^{40} \mathrm{Ar} /{ }^{39} \mathrm{Ar}$ isotopic age of $198 \pm 2 \mathrm{Ma}$, which suggests that the mineralisation took place during the Triassic Indosinian Orogeny [39]. Implications of this are that the Neoproterozoic (ca. $927 \mathrm{Ma}$ ) magma-hydrothermal event associated with the emplacement of ultramafic intrusions. Triassic (ca. $216 \mathrm{Ma}$ ) porphyritic granites in the area are 18 Ma older than the Jianchaling mineralisation, again indicating that the granites are not related to the gold mineralisation.

The $\mathrm{C}, \mathrm{H}$ and $\mathrm{O}$ stable isotopes indicate that the ore-forming fluids are predominantly metamorphosed in origin and contaminated with meteoric fluid during the deposition of the gold at Jianchaling. On the other words, the mineralising fluid is probably sourced from the metamorphic dehydration of carbonate rocks in the Duantouya Formation, and gradually mixed with meteoric water. The ${ }^{87} \mathrm{Sr} /{ }^{86} \mathrm{Sr}, \mathrm{I}_{\mathrm{Sr}}(\mathrm{t}), \varepsilon_{\mathrm{Nd}}, \mathrm{Pb}$ and $\mathrm{S}$ isotopic data (Figure 10) confirm that the sulfides in the mineralised are sourced from the metamorphosed Duantouya Formation, Bikou Group and minor ultramafic rocks.

From the discussion above, combining the comparison of the geological characteristics between Jianchaling and Orogenic gold deposit (Table 7), the Jianchaling deposit is here interpreted as an orogenic-type deposit and summarised in Figure 11.

Table 7. Geological characteristics comparison between the Jianchaling and Orogenic gold deposit.

\begin{tabular}{|c|c|c|}
\hline & Orogenic Gold Deposit ${ }^{a}$ & Jianchaling Gold Deposit ${ }^{\text {b }}$ \\
\hline Tectonic setting & $\begin{array}{l}\text { Subduction hyperplasia, } \\
\text { continental collision, } \\
\text { intracontinental strike-slip and } \\
\text { intracontinental compressional } \\
\text { orogenic belt }\end{array}$ & Continental collision \\
\hline Ore-bearing terrane & Metamorphic terrane & $\begin{array}{l}\text { Metamorphic and sedimentary } \\
\text { terrane }\end{array}$ \\
\hline Ore-controlling lithology & $\begin{array}{l}\text { Ultramafic volcanic rocks, } \\
\text { intrusive rocks, miscellaneous } \\
\text { sandstones, slate }\end{array}$ & Ultramafic and dolomite \\
\hline Metamorphism of the host rock & $\begin{array}{l}\text { Greenschist facies (low green } \\
\text { schist to low granulite) }\end{array}$ & $\begin{array}{l}\text { Greenschist to low amphibolite } \\
\text { facies }\end{array}$ \\
\hline Ore-controlling structures & $\begin{array}{l}\text { In the secondary or lower faults of } \\
\text { the super-lithospheric fracture } \\
\text { zone, the ore-forming structures is } \\
\text { mainly the high angle oblique slip } \\
\text { belt, the reverse overthrust belt, } \\
\text { and also the transverse fracture, } \\
\text { the ductile-brittle zone }\end{array}$ & $\begin{array}{l}\text { In the brittle fracture of the ductile } \\
\text { shear zone (high angle thrust belt) } \\
\text { in the basement fault zone }\end{array}$ \\
\hline Ore and gangue minerals & Mostly pyrite & $\begin{array}{c}\text { Arsenic pyrite, marcasite, } \\
\text { arsenopyrite, orpiment, realgar, } \\
\text { quartz and calcite }\end{array}$ \\
\hline Ore type & Quartz vein and altered rock & Altered rock \\
\hline
\end{tabular}


Table 7. Cont.

\begin{tabular}{|c|c|c|}
\hline & Orogenic Gold Deposit ${ }^{a}$ & Jianchaling Gold Deposit ${ }^{\text {b }}$ \\
\hline $\begin{array}{l}\text { Metallogenic hydrothermal fluids } \\
\text { characteristics }\end{array}$ & $\begin{array}{c}\text { Aqueous solution with low } \\
\text { salinity and low density, } \\
\text { containing } \mathrm{CO}_{2} \pm \mathrm{CH}_{4} \pm \mathrm{N}_{2} \pm \\
\mathrm{H}_{2} \mathrm{~S} \text {. The fluids inclusion type has } \\
\mathrm{H}_{2} \mathrm{O}-\mathrm{CO}_{2} \text {, rich } \mathrm{CO}_{2} \text { (with an } \\
\text { unquantifiable } \mathrm{CH}_{4} \text { and a small } \\
\text { amount of } \mathrm{H}_{2} \mathrm{O} \text { ) and gas-liquid } \\
\text { two-phase } \mathrm{H}_{2} \mathrm{O} \text { inclusions }\end{array}$ & $\begin{array}{l}\text { Aqueous solution with low } \\
\text { salinity and low density, } \\
\text { containing } \mathrm{CO}_{2} \pm \mathrm{CH}_{4} \pm \mathrm{H}_{2} \mathrm{~S} \text {. } \\
\text { The fluids inclusion type has } \\
\mathrm{NaCl}-\mathrm{H}_{2} \mathrm{O}, \mathrm{CO}_{2}-\mathrm{H}_{2} \mathrm{O}-\mathrm{NaCl} \pm \\
\mathrm{CH}_{4} \text { and pure } \mathrm{CO}_{2}-\mathrm{CH}_{4}\end{array}$ \\
\hline Metallogenic fluids salinity & $3-12$ wt $\% \mathrm{NaCl}$ equiv. & $0.4-15.6$ wt $\% \mathrm{NaCl}$. equiv. \\
\hline Main metallogenic temperature & $350 \pm 50^{\circ} \mathrm{C}$ & $200-320^{\circ} \mathrm{C}$ \\
\hline Mineralization pressure & $50-400 \mathrm{MPa}$ & $117-354 \mathrm{MPa}$ \\
\hline Initial metallogenic fluids feature & Metamorphic fluids & Metamorphic fluids \\
\hline
\end{tabular}

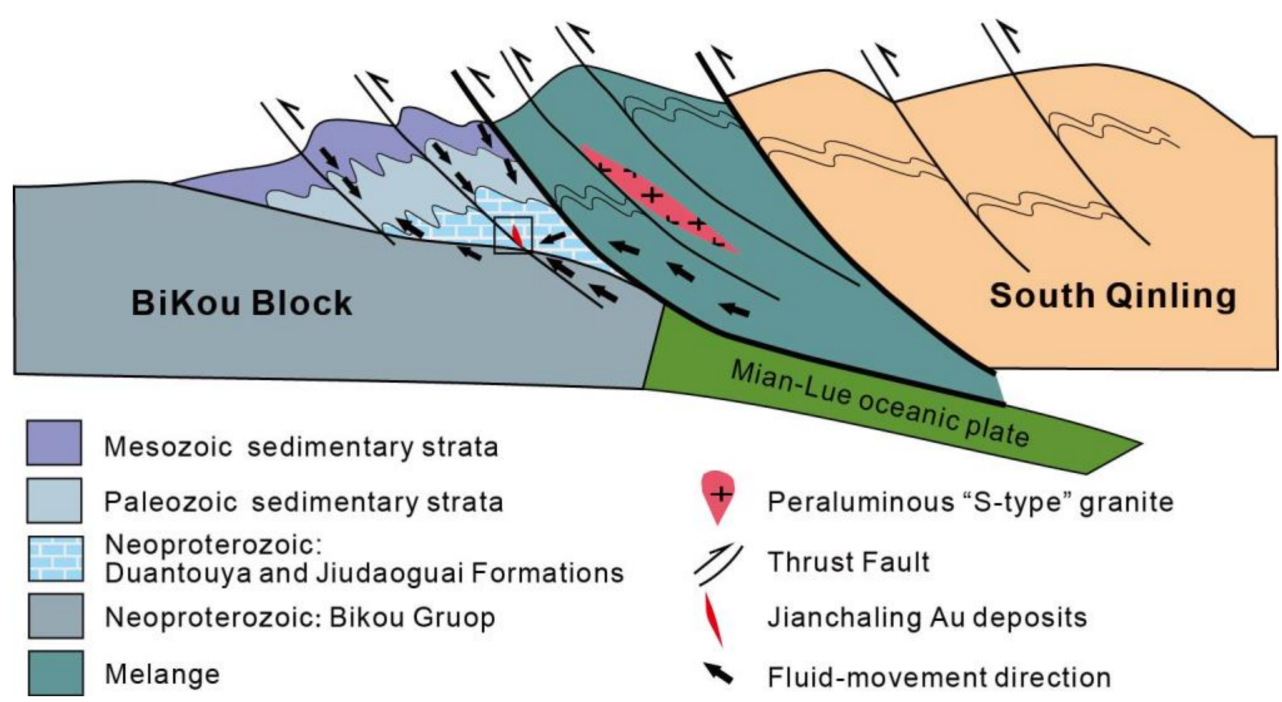

Figure 11. Tectonic model for the Jianchaling Au deposit [39].

\section{Conclusions}

Hydrogen, oxygen and carbon isotopic systematics indicate that the ore-forming fluids at the Jianchaling Au deposit progressively evolved from an early stage represented by the first vein set associated with deformation and metamorphism of the country rocks. This was succeeded by a late tectonic stage represented by the mineralised second vein set sourced from a mixed metamorphic and meteoric fluid, and progressed to a late stage represented by the third vein set. The S-Pb-Sr-Nd isotopic data indicate that the mineralising fluids were sourced locally around Jianchaling. From these observations, the Jianchaling Au deposit is interpreted as an orogenic Au deposit formed during the Mesozoic Indosinian Orogeny.

Acknowledgments: The National Natural Science Foundation (Numbers 41403032, 41502070 and 41202050), and the National Crisis Mine Prospecting Foundation (Number 20089934) financially supports this study. We are grateful to Wenping Zhu for help during isotope analyses and the Exploration Team '711' of Northwest Mining and Geology Group Co., Ltd. for their help during field. Yanjing Chen, Huayong Chen and three anonymous reviewers are thanked for their constructive suggestions, which have greatly improved this manuscript. 
Author Contributions: Su-Wei Yue and Zhen-Wen Lin participated in field samples collection; Su-Wei Yue conceived and designed the experiments; Su-Wei Yue and Jing Fang performed the experiments; Deng-Feng Li and Leon Bagas analyzed the data; Su-Wei Yue wrote the paper, assisted by all other authors.

Conflicts of Interest: The authors declare no conflict of interest.

\section{References}

1. Chen, Y.J.; Zhai, M.G.; Jiang, S.Y. Significant achievements and open issues in study of orogenesis and metallogenesis surrounding the North China continent. Acta Petrol. Sin. 2009, 25, 2695-2726.

2. Chen, Y.J.; Santosh, M.; Somerville, I.; Chen, H.Y. Indosinian tectonics and mineral systems in China: An introduction. Geol. J. 2014, 49, 331-337. [CrossRef]

3. Chen, Y.J. The development of continental collision metallogeny and its application. Acta Petrol. Sin. 2013, 29, 1-17. [CrossRef]

4. Groves, D.I.; Goldfarb, R.J.; Gebre-Mariam, M.; Hagemann, S.G.; Robert, F. Orogenic gold deposits: A proposed classification in the context of their crustal distribution and relationship to other gold deposit types. Ore Geol. Rev. 1998, 13, 7-27. [CrossRef]

5. Goldfarb, R.J.; Groves, D.I.; Gardoll, S. Orogenic gold and geologic time: A global synthesis. Ore Geol. Rev. 2001, 18, 1-75. [CrossRef]

6. Goldfarb, R.; Baker, T.; Dube, B.; Groves, D.I.; Hart, C.J.R.; Gosselin, P. Distribution, character and genesis of gold deposits in metamorphic terranes. Soc. Econ. Geol. 2005, 407-450. [CrossRef]

7. Chen, Y.J.; Ni, P.; Fan, H.R.; Pirajno, F.; Lai, Y.; Su, W.C.; Zhang, H. Diagnostic fluid inclusions of different types hydrothermal gold deposits. Acta Petrol. Sin. 2007, 23, 2085-2108.

8. Mao, J.W.; Qiu, Y.M.; Goldfarb, R.; Zhang, Z.C.; Garwin, S.; Ren, F.S. Geology, distribution, and classification of gold deposits in the western Qinling belt, central China. Miner. Depos. 2002, 37, 352-377. [CrossRef]

9. Chen, Y.J.; Santosh, M. Triassic tectonics and mineral systems in the Qinling Orogen, central China. Geol. J. 2014, 49, 338-358. [CrossRef]

10. Goldfarb, R.J.; Groves, D.I. Orogenic gold: Common or evolving fluid and metal sources through time. Lithos 2015, 233, 2-26. [CrossRef]

11. Chang, Z.; Large, R.R.; Maslennikov, V. Sulfur isotopes in sediment-hosted orogenic gold deposits: Evidence for an early timing and a seawater sulfur source. Geology 2008, 36, 971-974. [CrossRef]

12. Bierlein, F.P.; Arne, D.C.; Cartwright, I. Stable isotope $(C, O, S)$ systematics in alteration haloes associatedwith orogenic gold mineralization in the Victorian gold province, SE Australia. Geochem. Explor. Environ. Anal. 2004, 4, 191-211. [CrossRef]

13. Groves, D.I.; Santosh, M. The giant Jiaodong gold province: The key to a unified model for orogenic gold deposits? Geosci. Front. 2016, 7, 409-417. [CrossRef]

14. Chen, Y.J.; Pirajno, F.; Qi, J.P.; Li, J.; Wang, H.H. Ore geology, fluid geochemistry and genesis of the Shanggong gold deposit, Eastern Qinling Orogen, China. Resour. Geol. 2006, 56, 99-116. [CrossRef]

15. Chen, Y.J.; Pirajno, F.; Sui, Y.H. Isotope geochemistry of the Tieluping silver-lead deposit, Henan, China: A case study of orogenic silver-dominated deposits and related tectonic setting. Miner. Depos. 2004, 39, 560-575. [CrossRef]

16. Chen, Y.J.; Pirajno, F.; Qi, J.P. The Shanggong gold deposit, Eastern Qinling Orogen, China: Isotope geochemistry and implications for ore genesis. J. Asian Earth Sci. 2008, 33, 252-266. [CrossRef]

17. Yao, J.M.; Zhao, T.P.; Wei, Q.G.; Yuan, Z.L. Fluid inclusion features and genetic type of the Wangpingxigou $\mathrm{Pb}-\mathrm{Zn}$ deposit, Henan Province. Acta Petrol. Sin. 2008, 24, 2113-2123.

18. Li, N.; Chen, Y.J.; Fletcher, I.R.; Zeng, Q.T. Triassic mineralization with Cretaceous overprint in the Dahu $\mathrm{Au}-\mathrm{Mo}$ deposit, Xiaoqinling gold province: Constraints from SHRIMP monazite U-Th-Pb geochronology. Gondwana Res. 2011, 20, 543-552. [CrossRef]

19. Zhang, J.; Chen, Y.J.; Yang, Y.; Deng, J. Lead isotope systematics of the Weishancheng Au-Ag belt, Tongbai Mountains, central China: Implication for ore genesis. Int. Geol. Rev. 2011, 53, 656-676. [CrossRef]

20. Ni, Z.Y.; Chen, Y.J.; Li, N.; Zhang, H. Pb-Sr-Nd isotope constraints on the fluid source of the Dahu Au-Mo deposit in Qinling Orogen, central China, and implication for Triassic tectonic setting. Ore Geol. Rev. 2012, 46, 60-67. [CrossRef] 
21. Yue, S.W.; Lin, Z.W.; Deng, X.H.; Li, F.R.; He, H.X.; Feng, A.G. C, H, O, S, Pb isotopic geochemistry of the Jianchaling gold deposit, Shaanxi Province. Geotecton. Metallog. 2013, 37, 653-670.

22. Deng, X.H.; Santosh, M.; Yao, J.M.; Chen, Y.J. Geology, fluid inclusions and sulphur isotopes of the Zhifang Mo deposit in Qinling Orogen, central China: A case study of orogenic-type Mo deposits. Geol. J. 2014, 49, 515-533. [CrossRef]

23. Deng, X.H.; Chen, Y.J.; Santosh, M.; Yao, J.M.; Sun, Y.L. Re-Os and Sr-Nd-Pb isotope constraints on source of fluids in the Zhifang Mo deposit, Qinling Orogen, China. Gondwana Res. 2016, 30, 132-143. [CrossRef]

24. Yue, S.W.; Deng, X.H.; Bagas, L. Geology, isotope geochemistry, and ore genesis of the Yindonggou Ag-Au (-Pb-Zn) deposit, Hubei Province, China. Geol. J. 2014, 49, 442-462. [CrossRef]

25. Zhou, Z.J.; Lin, Z.W.; Qin, Y. Geology, geochemistry and genesis of the Huachanggou gold deposit, western Qinling Orogen, central China. Geol. J. 2014, 49, 424-441. [CrossRef]

26. Lin, Z.W.; Zhou, Y.Z.; Qin, Y.; Yue, S.W. Fuchsite ${ }^{40} \mathrm{Ar} /{ }^{39} \mathrm{Ar}$ Geochronology of the Huachanggou Gold Deposit and its Tectonic Implications. Geotecton. Metallog. 2017, 41, 315-324. [CrossRef]

27. Chen, Y.J.; Pirajno, F.; Li, N.; Guo, D.S.; Lai, Y. Isotope systematics and fluid inclusion studies of the Qiyugou breccia pipe-hosted gold deposit, Qinling Orogen, Henan province, China: Implications for ore genesis. Ore Geol. Rev. 2009, 35, 245-261. [CrossRef]

28. Chen, Y.J.; Wang, Y. Fluid inclusion study of the Tangjiaping Mo deposit, Dabie Shan, Henan Province: Implications for the nature of the porphyry systems of post-collisional tectonic settings. Int. Geol. Rev. 2011, 53, 635-655. [CrossRef]

29. Fan, H.R.; Hu, F.F.; Wilde, S.A.; Yang, K.F.; Jin, C.W. The Qiyugou gold-bearing breccia pipes, Xiong'ershan region, central China: Fluid-inclusion and stable-isotope evidence for an origin from magmatic fluids. Int. Geol. Rev. 2011, 53, 25-45. [CrossRef]

30. Li, N.; Chen, Y.J.; Pirajno, F.; Gong, H.J.; Mao, S.D.; Ni, Z.Y. LA-ICP-MS zircon U-Pb dating, trace element and Hf isotope geochemistry of the Heyu granite batholith, eastern Qinling, central China: Implications for Mesozoic tectono-magmatic evolution. Lithos 2012, 142-143, 34-47. [CrossRef]

31. Li, N.; Chen, Y.J.; Pirajno, F.; Ni, Z.Y. Timing of the Yuchiling giant porphyry Mo system, and implications for ore genesis. Miner. Depos. 2013, 48, 505-524. [CrossRef]

32. Yang, Y.F.; Li, N.; Chen, Y.J. Fluid inclusion study of the Nannihu giant porphyry Mo-W deposit, Henan Province, China: Implications for the nature of porphyry ore-fluid systems formed in a continental collision setting. Ore Geol. Rev. 2012, 46, 83-94. [CrossRef]

33. Yang, Y.F.; Chen, Y.J.; Li, N.; Mi, M.; Xu, Y.L.; Li, F.L.; Wan, S.Q. Fluid inclusion and isotope geochemistry of the Qian'echong giant porphyry Mo deposit, Dabie Shan, China: A case of $\mathrm{NaCl}$-poor, $\mathrm{CO}_{2}$-rich fluid systems. J. Geochem. Explor. 2013, 124, 1-13. [CrossRef]

34. Deng, X.H.; Chen, Y.J.; Santosh, M.; Zhao, G.C.; Yao, J.M. Metallogeny during continental outgrowth in the Columbia supercontinent: Isotopic characterization of the Zhaiwa Mo-Cu system in the North China Craton. Ore Geol. Rev. 2013, 51, 43-56. [CrossRef]

35. Deng, X.H.; Chen, Y.J.; Santosh, M.; Yao, J.M. Re-Os geochronology, fluid inclusions and genesis of the 0.85Ga Tumen molybdenite-fluorite deposit in Eastern Qinling, China: Implications for pre-Mesozoic Mo enrichment and tectonic setting. Geol. J. 2013, 48, 484-497. [CrossRef]

36. Mao, S.D.; Chen, Y.J.; Zhou, Z.J.; Lu, Y.H.; Guo, J.H.; Qin, Y.; Yu, J.Y. Zircon geochronology and Hf isotope geochemistry of the granitoids in the Yangshan gold field, western Qinling, China: Implications for petrogenesis, ore genesis and tectonic setting. Geol. J. 2014, 49, 359-382. [CrossRef]

37. Zeng, Q.T.; Mccuaig, T.C.; Tohver, E.; Bagas, L.; Lu, Y. Episodic Triassic magmatism in the western South Qinling Orogen, central China, and its implications. Geol. J. 2014, 49, 402-423. [CrossRef]

38. Zhang, Y.; Tang, H.S.; Chen, Y.J.; Leng, C.B.; Zhao, C.H. Ore geology, fluid inclusion and isotope geochemistry of the Xunyang Hg-Sb orefield, Qinling Orogen, Central China. Geol. J. 2014, 49, 463-481. [CrossRef]

39. Yue, S.W.; Deng, X.H.; Bagas, L.; Lin, Z.W.; Fang, J.; Zhu, C.H.; Zhang, W. Fluid inclusion geochemistry and ${ }^{40} \mathrm{Ar} /{ }^{39} \mathrm{Ar}$ geochronology constraints on the genesis of the Jianchaling Au deposit, China. Ore Geol. Rev. 2017, 80, 676-690. [CrossRef]

40. Zhang, F.X.; Wang, J.Y. The genetic relationship between the ultrabasic rock and gold deposit in Jianchaling, Shaanxi. Gold Geol. 1999, 5, 14-20.

41. Ma, J.Q. Qinling Orogenic Belt Mianluening Gold Deposit Formation Mode and Prospecting Direction; Institute of Geochemistry (Guiyang), Chinese Academy of Sciences: Beijing, China, 1998. 
42. Ren, X.H. Research on Metalliferous Deposit Mineralization and Survey and Prediction of Target Area for Mineral Prospecting Mian-Lue-Ning Region, Shannxi; Chang'an University: Xi'an, China, 2008.

43. Wang, R.T.; Wang, D.S.; Li, F.R.; Chen, L.X.; Dai, J.Z.; Wang, Y.T.; Yan, Z. Geochemical characteristics, metallogenic geodynamics and prospecting indicator of the Jianchaling Large Gold Ore Deposit. Acta Geol. Sin. 2009, 83, 1739-1751.

44. Chen, Y.J.; Fu, S.G. Gold Mineralization in West Henan; Seismological Press: Beijing, China, 1992, ISBN 9787502808013.

45. Zhang, G.W.; Meng, Q.R.; Yu, Z.P.; Sun, Y.; Zhou, D.W.; Guo, A.L. Orogenesis and dynamics of the Qinling orogen. Sci. China Ser. D Earth Sci. 1996, 26, 193-200.

46. Chen, Y.J. Indosinian tectonic setting, magmatism and metallogenesis in Qinling Orogen, central China. Geol. China 2010, 37, 854-865.

47. Gao, S. Geochemical evidence for the Proterozoic tectonic evolution of the Qinling Orogenic Belt and its adjacent margins of the North China and Yangtze cratons. Precambrian Res. 1996, 80, 23-48. [CrossRef]

48. Dong, Y.P.; Zhang, G.W.; Neubauer, F.; Liu, X.M.; Genser, J.; Hauzenberger, C. Tectonic evolution of the Qinling orogen, China: Review and synthesis. J. Asian Earth Sci. 2011, 41, 213-237. [CrossRef]

49. Li, N.; Chen, Y.J.; Santosh, M.; Pirajno, F. Compositional polarity of Triassic granitoids in the Qinling Orogen, China: Implication for termination of the northernmost paleo-Tethys. Gondwana Res. 2015, 27, 244-257. [CrossRef]

50. Jiang, X.D.; Wei, G.F.; Nie, J.T. Jianchaling nickel deposit: Magmatic or hydrothermal origin. Miner. Depos. 2010, 29, 1112-1124.

51. Hu, J.M.; Dong, G.F. Rule of spatial extention and direction in prospecting of the Jianchaling gold deposit in Lueyang County. Geotecton. Metallog. 2002, 26, 75-80.

52. Tanaka, T.; Togashi, S.; Kamioka, H.; Amakawa, H.; Kagami, H.; Hamamoto, T.; Yuhara, M.; Orihashi, Y.; Yoneda, S.; Shimizu, H.; et al. JNdi-1: A neodymium isotopic reference in consistency with LaJolla neodymium. Chem. Geol. 2000, 168, 279-281. [CrossRef]

53. Yuan, L.X.; Zhang, M.P.; Hou, J.F.; Qin, T.T. Characteristics of mass components and metallogenic fluids of the Maoergou gold deposit in Lueyang County of Shaanxi Province. Geol. Shaanxi 2004, 22, 25-34.

54. Clayton, R.N.; O'Neil, J.R.; Mayeda, T.K. Oxygen isotope exchange between quartz and water. J. Geophys. Res. 1972, 77, 3057-3067. [CrossRef]

55. Zheng, Y.F. Oxygen isotope fractionation in carbonate and sulfate minerals. Geochem. J. 1999, 33, 109-126. [CrossRef]

56. Sheppard, S.M.F.; Schwarcz, H.P. Fractionation of carbon and oxygen isotopes and magnesium between coexisting metamorphic calcite and dolomite. Contrib. Mineral. Petrol. 1970, 26, 161-198. [CrossRef]

57. Chacko, T.; Mayeda, T.K.; Clayton, R.N.; Goldsmith, J.R. Oxygen and carbon isotope fractionations between $\mathrm{CO}_{2}$ and calcite. Geochim. Cosmochim. Acta 1991, 55, 2867-2882. [CrossRef]

58. Huang, J.J.; Huang, B.; Ren, X.H.; Xu, W.X.; Liao, J.H. The application of Lead isotope tracerlocation for Jianchaling gold deposit. Gold Sci. Technol. 2002, 10, 33-40.

59. Zhou, Z.J. Comparative Study of the Huachanggou Gold Deposit in Southern Qinling and the Wenyu Gold Deposit in Xiaoqinling Terrane; University of Chinese Academy of Sciences: Beijing, China, 2012.

60. Li, Y.F.; Lai, S.C.; Qin, J.F.; Liu, X.; Wang, J. Geochemistry and Sr-Nd-Pb isotopic composition of Bikou Group volcanic rocks: Evidence of North Yangtze splitting. Sci. China Ser. D Earth Sci. 2007, 37, 295-306.

61. Yan, Q.R.; Andrew, D.H.; Wang, Z.Q.; Yan, Z.; Peter, A.D.; Wang, T.; Liu, D.Y.; Song, B.; Jiang, C.F. Geochemistry and tectonic setting of the Bikou volcanic terrane on the northern margin of the Yangtze plate. Acta Petrol. Mineral. 2004, 23, 1-11.

62. Hoefs, J. Stable Isotope Geochemistry, 6th ed.; Springer: Berlin, Germany, 2009; Volume 6, ISBN 9783540707035.

63. Kerrich, R.; Goldfarb, R.; Groves, D.; Garwin, S.; Jia, Y.F. The characteristics, origins, and geodynamic settings of supergiant gold metallogenic provinces. Sci. China Ser. D Earth Sci. 2000, 43, 1-68. [CrossRef]

64. Pang, J.L.; Qiu, Y.Z.; Liu, Y. Roles played by Ultra-basic rock in metallogeny of Jianchaling Au deposit. J. Chin. Rare Earth Soc. 1994, 9, 59-65.

65. Zheng, C.Y.; Liu, J.D.; Yuan, B.; Chen, S.J.; Dong, G.F.; Zhang, X.L.; Song, X.H. Geological and geochemical characteristics of rock mass related with gold mineralization in the Jianchaling deposit. Geol. Prospect. 2007, $43,52-57$.

66. Zhang, J.; Chen, Y.J.; Pirajno, F.; Deng, J.; Chen, H.Y.; Wang, C.M. Geology, C-H-O-S-Pb isotope systematics and geochronology of the Yindongpo gold deposit, Tongbai Mountains, Central China: Implication for ore genesis. Ore Geol. Rev. 2013, 53, 343-356. [CrossRef] 
67. Pang, C.Y.; Chen, M.Y. Isotopic geochronological data and their geological significance in Jianchaling region, Shaanxi Province. Miner. Resour. Geol. 1993, 7, 65-71.

68. Huang, W.; Ran, H. The Metallogenetic Characteristics of Gold Deposits Related to Carbonatized Ultramafites in Ophiolites. Bull. Mineral. Petrol. Geochem. 1996, 15, 153-156.

69. Jiang, S.Y.; Dai, B.Z.; Jiang, Y.H.; Zhao, H.X.; Hou, M.L. Jiaodong and Xiaoqinling: Two orogenic gold provinces formed in different tectonic settings. Acta Petrol. Sin. 2009, 25, 2727-2738.

70. Goldfarb, R.J.; Santosh, M. The dilemma of the Jiaodong gold deposits: Are they unique? Geosci. Front. 2014, 5, 139-153. [CrossRef]

71. Taylor, H.P. The application of oxygen and hydrogen isotope studies to problems of hydrothermal alteration and ore deposition. Econ. Geol. 1974, 69, 843-883. [CrossRef]

72. Hoefs, J. Stable Isotope Geochemistry, 4th ed.; Springer: Berlin, Germany, 1997, ISBN 3540707034.

73. Faure, G. Principles of Isotope Geology, 2nd ed.; Wiley: New York, NY, USA, 1986, ISBN 9780471864127.

74. Guerrera, J.A.; Peacock, S.M.; Knauth, L.P. Large $18 \mathrm{O}$ and $13 \mathrm{C}$ depletions in greenschist facies carbonate rocks, western Arizona. Geology 1997, 25, 943-946. [CrossRef]

75. Ohmoto, H.; Goldhaber, M.B. Sulfur and carbon isotopes. In Geochemistry of Hydrothermal Ore Deposits; John Wiley \& Sons: New York, NY, USA, 1997; Volume 3, pp. 517-611.

76. Chen, H.Y.; Chen, Y.J.; Baker, M. Isotopic geochemistry of the Sawayaerdun orogenic-type gold deposit, Tianshan, northwest China: Implications for ore genesis and mineral exploration. Chem. Geol. 2012, 310, 1-11. [CrossRef]

77. Zhao, R. Sulfur isotope study of some endogenic ore deposits in Hebei, Shandong and Anhui Provinces. Acta Petrol. Sin. 1986, 2, 26-32.

78. Li, W.B.; Huang, Z.L.; Zhang, G. Sources of the ore metals of the Huize ore field in Yunnan Province:constraints from $\mathrm{Pb}, \mathrm{S}, \mathrm{C}, \mathrm{H}, \mathrm{O}$ and Sr isotope geochemistry. Acta Petrol. Sin. 2006, 22, 2567-2580.

79. Gavrielli, I.; Starinsky, A.; Spiro, B.; Aizenshtat, Z.; Nielsen, H. Mechanisms of sulfate removal from subsurface calcium chloride brines: Heletz-Kokhav oilfields, Israel. Geochim. Cosmochim. Acta 1995, 59, 3525-3533. [CrossRef]

80. Goldfarb, R.J.; Newberry, R.J.; Pickthorn, W.J.; Gent, C.A. Oxygen, hydrogen, and sulfur isotope studies in the Juneau gold belt, southeastern Alaska: Constraints on the origin of hydrothermal fluids. Econ. Geol. 1991, 86, 66-80. [CrossRef]

81. Jia, Y.F.; Kerrich, R.; Goldfarb, R. Metamorphic Origin of Ore-Forming Fluids for Orogenic Gold-Bearing Quartz Vein Systems in the North American Cordillera: Constraints from a Reconnaissance Study of $\delta 15 \mathrm{~N}$, $\delta$ D, and $\delta 18 \mathrm{O}$. Econ. Geol. 2003, 98, 109-123. [CrossRef]

82. Zhang, J.; Yang, Y.; Lu, Y.H.; Chen, Y.J.; Wan, S.Q.; Ma, H.W. Geological and geochemical characteristics and genesis of the Poshan silver deposit, Henan Province. Geol. China 2008, 35, 1220-1229.

83. Zartman, R.E.; Haines, S.M. The plumbotectonic model for Pb isotopic systematics among major terrestrial reservoirs-A case for bi-directional transport. Geochim. Cosmochim. Acta 1988, 52, 1327-1339. [CrossRef]

84. Chappell, B.W.; White, A.J.R. Two contrasting granite types. Pac. Geol. 1974, 8, 173-174. [CrossRef]

85. Chen, J.; Jahn, B. Crustal evolution of southeastern China: Nd and Sr isotopic evidence. Tectonophysics 1998, 284, 101-133. [CrossRef]

86. Wang, X.C.; Li, X.H.; Li, W.X.; Li, Z.X.; Liu, Y.; Yang, Y.H.; Liang, X.R.; Tu, X.L. The Bikou basalts in the northwestern Yangtze block, South China: Remnants of 820-810 Ma continental flood basalts? Bull. Geol. Soc. Am. 2008, 120, 1478-1492. [CrossRef]

87. Groves, D.I.; Condie, K.C.; Goldfarb, R.J.; Hronsky, J.M.A.; Vielreicher, R.M. Secular changes in global tectonic processes and their influence on the temporal distribution of gold-bearing mineral deposits. Econ. Geol. 2005, 100, 203-224. [CrossRef]

88. Chen, Y.J. Orogenic-type deposits and their metallogenic model and exploration potential. Geol. China 2006, 33, 1181-1196.

(C) 2018 by the authors. Licensee MDPI, Basel, Switzerland. This article is an open access article distributed under the terms and conditions of the Creative Commons Attribution (CC BY) license (http:/ / creativecommons.org/licenses/by/4.0/). 